\title{
Information metric, Berry connection, and Berezin-Toeplitz quantization for matrix geometry
}

\author{
Goro Ishiki, ${ }^{1,2, *}$ Takaki Matsumoto, ${ }^{2, \dagger}$ and Hisayoshi Muraki ${ }^{3, \$}$ \\ ${ }^{1}$ Tomonaga Center for the History of the Universe, University of Tsukuba, \\ Tsukuba, Ibaraki 305-8571, Japan \\ ${ }^{2}$ Graduate School of Pure and Applied Sciences, University of Tsukuba, Tsukuba, Ibaraki 305-8571, Japan \\ ${ }^{3}$ Department of Physics, Sogang University, Seoul 04107, Korea
}

(Received 3 May 2018; published 3 July 2018)

\begin{abstract}
We consider the information metric and Berry connection in the context of noncommutative matrix geometry. We propose that these objects give a new method of characterizing the fuzzy geometry of matrices. We first give formal definitions of these geometric objects and then explicitly calculate them for the well-known matrix configurations of fuzzy $S^{2}$ and fuzzy $S^{4}$. We find that the information metrics are given by the usual round metrics for both examples, while the Berry connections coincide with the configurations of the Wu-Yang monopole and the Yang monopole for fuzzy $S^{2}$ and fuzzy $S^{4}$, respectively. Then, we demonstrate that the matrix configurations of fuzzy $S^{n}(n=2,4)$ can be understood as images of the embedding functions $S^{n} \rightarrow \mathbf{R}^{n+1}$ under the Berezin-Toeplitz quantization map. Based on this result, we also obtain a mapping rule for the Laplacian on fuzzy $S^{4}$.
\end{abstract}

DOI: $10.1103 /$ PhysRevD.98.026002

\section{INTRODUCTION}

In the matrix models for string and M- theories [1,2], geometry of fundamental objects such as strings and membranes are described in terms of some Hermitian matrices $X^{\mu}$, which correspond to the quantized version of the embedding functions. The quantization process to obtain the matrices is very similar to the canonical quantization of classical mechanical systems, in which coordinates and conjugate momenta are promoted to noncommutative operators acting on a Hilbert space. In the case of the matrix models, the noncommutativity is introduced purely between coordinates and this leads to the notion of the noncommutative matrix geometry.

A nice framework of this quantization process is given by the matrix regularization $[3,4]$. The matrix regularization can be defined for any compact symplectic manifold $(\mathcal{M}, \omega)$ and is characterized by a sequence $\left\{T_{N}\right\}$, where $N$ runs over an infinite set of strictly increasing positive integers and $T_{N}$ is a linear map from

\footnotetext{
*ishiki@het.ph.tsukuba.ac.jp

matsumoto@het.ph.tsukuba.ac.jp

"hmuraki@sogang.ac.kr
}

Published by the American Physical Society under the terms of the Creative Commons Attribution 4.0 International license. Further distribution of this work must maintain attribution to the author(s) and the published article's title, journal citation, and DOI. Funded by SCOAP. functions on $\mathcal{M}$ to $N \times N$ matrices. Basically, $T_{N}$ is required to satisfy

$$
\begin{aligned}
\left\|T_{N}(f) T_{N}(g)-T_{N}(f g)\right\| & \rightarrow 0, \\
\left\|i c_{N}\left[T_{N}(f), T_{N}(g)\right]-T_{N}(\{f, g\})\right\| & \rightarrow 0, \\
\operatorname{Tr} T_{N}(f) & \rightarrow \int \omega^{2 n} f,
\end{aligned}
$$

as $N \rightarrow \infty$. Here, $\|\cdot\|$ is a matrix norm, $\{$,$\} is a Poisson$ bracket on $\mathcal{M}$ and $c_{N}$ is an $N$-dependent constant, which goes to infinity as $N \rightarrow \infty$ and controls the magnitude of noncommutativity. The first condition in (1.1) says that the algebra of matrices approximates the algebra of functions. In particular, it implies that the matrices $T_{N}(f)$ become commutative in the large- $N$ limit. The second condition means that, in the large- $N$ limit, the Poisson algebra can also be well-approximated by the commutator algebra of matrices. The third condition for integrals can be used to map action functionals on $\mathcal{M}$ to matrix models.

Though most well-known matrix geometries such as the fuzzy $C P^{n}$, fuzzy tori and so on [5-8] can be regarded as concrete examples of the matrix regularization, there are some other examples which do not fit into the definition of the matrix regularization. In particular, since the definition of the matrix regularization depends on the symplectic structure, it can not be applied to nonsymplectic manifolds. For example, $S^{4}$ is not a symplectic manifold and its fuzzy version [9] gives a typical example which can not be 
described naively as the matrix regularization of foursphere. ${ }^{1}$ The fact that these nonsymplectic spaces also play important roles in understanding D-branes in the matrix models [9] suggests that the requirements of the matrix regularization (1.1) may be too strong, and more fundamental framework may be necessary to understand fuzzy geometries in the matrix models.

In this paper, we consider the Berezin-Toeplitz quantization for spinor bundles [11,12]. This method can be defined on a compact Riemannian spin-C manifold equipped with a topologically nontrivial gauge field configuration. This method provides a systematic way of generating a linear map from functions on the manifold to $N \times N$ matrices. Here, the space of $N \times N$ matrices arises as a restriction of the functional space of spinors to the space of Dirac zero modes, where $N$ is the dimension of the kernel of the Dirac operator and is related to the topological charge (such as the monopole charge or instanton number) of the gauge field by the index theorem. For Kähler manifolds, this mapping has been shown to satisfy the properties (1.1) of the matrix regularization, as a consequence of the Kähler compatibility condition $[11,12]$. However, since the definition of this quantization depends only on the metric and gauge connection, the BerezinToeplitz quantization map can be defined for nonsymplectic manifolds as well. Therefore, the Berezin-Toeplitz quantization may serve as a more fundamental mathematical framework for matrix models. Though a lot of concrete matrix configurations corresponding to various objects in string/M theories have been explicitly constructed so far [5-8], to our best knowledge, little work has been done to clarify the connection between those configurations and the Berezin-Toeplitz quantization scheme. ${ }^{2}$ In this paper, we try to understand this connection more deeply to see whether this quantization method indeed gives a good framework for matrix models or not.

The problem we consider in this paper is an inverse problem of the construction of the Berezin-Toeplitz quantization. In the Berezin-Toeplitz quantization, matrices (Toeplitz operators) are obtained from continuous geometric data, such as manifolds and Dirac zero modes. ${ }^{3}$ Conversely, in this paper, we try to extract the geometric data from a given set of Hermitian matrices $X^{\mu}$ which define a fuzzy space. This problem should be particularly important in studying the matrix models, which are formulated completely in the language of matrices.

The most important geometric data in the BerezinToeplitz quantization are the metric and the gauge

\footnotetext{
${ }^{1}$ See [10] and references therein for various descriptions of fuzzy $S^{4}$.

${ }^{2}$ This quantization has been studied in terms of the lowest Landau level problem on some monopole backgrounds. See [13-15].

${ }^{3}$ More precisely, we mean by the geometric data the triplet of the manifold, the metric and the gauge connection. Note that the Dirac zero modes can be constructed from them.
}

connection. In order to recover these geometric objects from the matrices $X^{\mu}$, we propose the use of the information metric and Berry connection. These objects are calculable from the matrices $X^{\mu}$ and also serve as new objects characterizing the geometry of the fuzzy spaces.

The definitions of the information metric and Berry connection are based on the notion of the coherent states in fuzzy spaces, which has been studied in various contexts recently. In [16], the coherent states were introduced for fuzzy spaces based on the viewpoint that they should have minimal wave packets in the target space in the large- $N$ limit. In this formulation, the coherent states are defined as ground states of a certain Hamiltonian. This construction was then generalized to the case of finite- $N$ matrices [17]. In the earlier work [18], the use of Dirac operator on D0branes was proposed based on a string-theory viewpoint. This method also leads to the notion of the coherent states. Since in all of these formulations, coherent states play very important roles, we call these methods collectively the coherent state methods in this paper. See [19-21] for some analysis using the coherent state methods. See also [22,23] for a nice interpretation of the coherent state methods in the system of non-Bogomol'nyi-Prasad-Sommerfield (BPS) D-branes.

Based on the coherent state methods, one can define the information metric and Berry connection for fuzzy spaces. In this paper, we first give formal definitions of these geometric objects. Then, we calculate the objects explicitly for fuzzy $S^{2}$ and fuzzy $S^{4}$ as examples. Finally, we show that for both cases the coherent states form a basis of the Dirac zero modes, so that the Hilbert space on which the matrices are acting can be identified with the space of the Dirac zero modes in the Berezin-Toeplitz quantization. We also demonstrate that, under the Berezin-Toeplitz quantization map, the defining Hermitian matrices for the fuzzy $S^{2}$ and $S^{4}$ can be seen as the images of the embedding functions of $S^{2}$ and $S^{4}$, respectively, into the flat target spaces. This result provides a unified viewpoint for fuzzy $S^{2}$ and fuzzy $S^{4}$. By using the quantization map, we also obtain explicit mapping rules for Laplacians on $S^{2}$ and $S^{4}$.

This paper is organized as follows. In Sec. II, we review the coherent state methods. In Sec. III, we introduce the information metric and Berry connection. In Sec. IV, we compute these structures for fuzzy $S^{2}$ and $S^{4}$. In Sec. V, we first review the Berezin-Toeplitz quantization and then show that the matrix configurations of fuzzy $S^{2}$ and fuzzy $S^{4}$ can be interpreted as the images of the embedding functions on $S^{2}$ and $S^{4}$, respectively. In Sec. VI, we summarize our results.

\section{COHERENT STATE METHODS}

For a given set of Hermitian matrices $\left\{X^{\mu}\right\}$, we can define an analogue of the coherent states. By using them, we can then associate the corresponding commutative space $\mathcal{M}$ with the given matrices. In this section, we 
review this process for two different methods based on the Hamiltonian and the Dirac operator.

\section{A. Hamiltonian}

We start with a set of $N \times N$ Hermitian matrices $\left\{X^{\mu}\right\}(\mu=1, \ldots, D)$, which defines a fuzzy space. We assume that there exists a commutative limit such that $X^{\mu}$ become mutually commuting and this limit is given by the large- $N$ limit. (For example, for the fuzzy sphere, $D=3$ and $X^{\mu}$ are given by the $N$-dimensional irreducible representation matrices $L_{i}(i=1,2,3)$ of the $S U(2)$ generators. For a unit sphere, $X^{i}$ should be normalized to satisfy $\left(X^{i}\right)^{2}=\mathbb{1}_{N}$ and thus $X^{i}=\frac{2}{\sqrt{N^{2}-1}} L_{i}$. With this normalization, $X^{i}$ become commuting matrices in the large- $N$ limit.) We also call this limit the classical limit by analogy with the quantum mechanics, where the commutative limit $\hbar \rightarrow 0$ indeed corresponds to the classical limit. In terms of the matrix regularization, this setup corresponds to a situation such that we are first given the images $X^{\mu}:=T_{N}\left(x^{\mu}\right)$ of the embedding functions $x^{\mu}: \mathcal{M} \rightarrow \mathbf{R}^{D}$. But the following arguments apply not only to the symplectic manifolds but also to nonsymplectic manifolds such as $S^{4}$. In the latter case, one can also construct the corresponding matrices $X^{\mu}$ based on the observations of D-brane charges or symmetries [9].

For the given $N \times N$ Hermitian matrices $\left\{X^{\mu}\right\}$, we first introduce the Hamiltonian, which is an $N \times N$ Hermitian matrix defined by

$$
H(y)=\frac{1}{2} \sum_{\mu=1}^{D}\left(X_{\mu}-y_{\mu} \mathbb{1}_{N}\right)^{2} .
$$

Here $y_{\mu}(\mu=1,2, \ldots, D)$ are real parameters and $\mathbb{1}_{N}$ stands for the $N \times N$ identity matrix. In the following, we omit the $N \times N$ identity matrix $\mathbb{1}_{N}$ for notational simplicity. Since the Hamiltonian $H(y)$ is Hermitian for any $y$, it is always possible to diagonalize $H(y)$ by using unitary similarity transformations. We introduce a basis, on which $H(y)$ becomes diagonal:

$$
H(y)|n, y\rangle=E_{n}(y)|n, y\rangle, \quad(n=0,1, \ldots, N-1) .
$$

Since $H(y)$ is a non-negative matrix, all the eigenvalues $E_{n}(y)$ are non-negative. We label the eigenvalues as $0 \leq E_{0}(y) \leq E_{1}(y) \leq \cdots \leq E_{N-1}(y)$. The eigenstates shall be normalized as $\langle n, y \mid m, y\rangle=\delta_{n m}$.

In quantum mechanics, the canonical coherent states are the states with minimal wave packets and in particular, the sizes of the wave packets go to zero in the classical limit $\hbar \rightarrow 0$. For fuzzy spaces defined by $\left\{X^{\mu}\right\}$, we can introduce an analogue of the canonical coherent states by using the above Hamiltonian as follows. From the definition of the eigenstates, the lowest eigenvalue $E_{0}(y)$ can be expressed as
$E_{0}(y)=\langle 0, y|H(y)| 0, y\rangle=\frac{1}{2}\left(\left\langle X_{\mu}\right\rangle-y_{\mu}\right)^{2}+\frac{1}{2}\left(\Delta X_{\mu}\right)^{2}$,

where

$$
\begin{gathered}
\left\langle X_{\mu}\right\rangle=\left\langle 0, y\left|X_{\mu}\right| 0, y\right\rangle, \\
\Delta X_{\mu}=\sqrt{\left\langle 0, y\left|X_{\mu}^{2}\right| 0, y\right\rangle-\left\langle 0, y\left|X_{\mu}\right| 0, y\right\rangle^{2}} .
\end{gathered}
$$

In terms of the wave packet of the ground state $|0, y\rangle,\left\langle X_{\mu}\right\rangle$ corresponds to the position of the center of the wave packet in the target space, while $\Delta X_{\mu}$ corresponds to the size of the wave packet in the $\mu$ direction. Now, suppose that $E_{0}(y)$ for a certain $y$ goes to zero in the classical (commutative) limit as $E_{0}(y) \rightarrow 0$. Then, since both terms in the right-hand side of (2.3) are squared and positive, we have

$$
\left\{\begin{array}{l}
\left\langle X_{\mu}\right\rangle-y_{\mu} \rightarrow 0, \\
\Delta X_{\mu} \rightarrow 0 .
\end{array}\right.
$$

for all $\mu$ simultaneously. This means that at the point $y$, there exists a wave packet which can shrink to zero size in the classical limit. Note that the inverse statement is also true, namely, if there is a state which (is not necessarily an eigenstate of $H(y)$ but) satisfies (2.6) for a certain point $y \in \mathbf{R}^{D}$, the ground state energy $E_{0}(y)$ is vanishing in the classical limit. Thus, the zero loci of $E_{0}(y)$ in $\mathbf{R}^{D}$ is equivalent to the subspace of $\mathbf{R}^{D}$ such that there can exist a wave packet which shrinks to a point in the classical limit. Such states are counter objects of the canonical coherent states in quantum mechanics. From this analogy, we call $|0, y\rangle$ coherent states here if it satisfies (2.6).

For the fuzzy space defined by $\left\{X^{\mu}\right\}$, we can associate a classical (commutative) manifold $\mathcal{M}$ as a hypersurface in $\mathbf{R}^{D}$ defined as a set of points on which there exist coherent states. In other words, $\mathcal{M}$ is given by the zero loci of the Hamiltonian:

$$
\mathcal{M}=\left\{y \in \mathbf{R}^{D} \mid f(y)=0\right\},
$$

where we introduced a function

$$
f(y)=\lim _{N \rightarrow \infty} E_{0}(y) .
$$

Note that, in most cases of finite-size matrices, exact zero modes of the Hamiltonian do not exist, and the classical space $\mathcal{M}$ can only be defined with the large- $N$ limit in this method. However, this method can be extended to finite- $N$ cases with the use of quasicoherent states [17]. Note also that even in the large- $N$ limit, if we consider general matrices for $X^{\mu}$, there are a lot of cases where $\mathcal{M}$ becomes an empty set. In order to have a non empty set, $\left\{X^{\mu}\right\}$ need to become commutative in the large- $N$ limit as $\left[X^{\mu}, X^{\nu}\right] \rightarrow 0$. 
In summary, we first introduced the coherent states as the ground state eigenvectors of the Hamiltonian (2.1) which have vanishing eigenvalues in the large- $N$ limit. Next, we defined a classical space $\mathcal{M}$ as a set of points in $\mathbf{R}^{D}$ on which there exists the coherent states.

\section{B. Dirac operator}

We next introduce another method based on a matrix Dirac-type operator $[18,22,23]$. While the method using Hamiltonian is based on the analogy with the quantum mechanics, the method using Dirac operator is based on some observations in string theories. Here, we first show the mathematical treatment of this method and then explain its physical implications in string theory.

The Dirac operator is defined from the given matrix $X^{\mu}$ as

$$
\not D(y)=\delta_{\mu \nu} \Gamma^{\mu} \otimes\left(X^{\nu}-y^{\nu} \mathbb{1}_{N}\right) .
$$

This is a $2^{[D / 2]} N \times 2^{[D / 2]} N$ Hermitian matrix, where $[D / 2]$ stands for the maximal integer less than or equal to $D / 2$. Here $\Gamma^{\mu}$ are $2^{[D / 2]} \times 2^{[D / 2]}$ matrix representations of the $D$-dimensional Euclidean Clifford algebra:

$$
\left\{\Gamma^{\mu}, \Gamma^{\nu}\right\}=2 \delta^{\mu \nu} 1_{2^{[D / 2]}} .
$$

The classical space $\mathcal{M}$ is defined as a hypersurface on which there exist zero modes of the Dirac operator as follows. Since the Dirac operator is Hermitian matrix, it has real eigenvalues. We denote eigenvalues and eigenstates as

$\not(y)|n, y\rangle=E_{n}(y)|n, y\rangle, \quad\left(n=0,1, \ldots, 2^{[D / 2]} N-1\right)$,

where we order the eigenvalues as $\left|E_{0}(y)\right| \leq\left|E_{1}(y)\right| \leq$ $\cdots \leq\left|E_{2^{[D / 2]} N-1}(y)\right|$ and the eigenstates shall be normalized as $\langle n, y \mid m, y\rangle=\delta_{n m}$. Note that $E_{n}(y)$ can also take negative values unlike the case of the Hamiltonian. The classical space $\mathcal{M}$ is defined as hypersurfaces on which zero modes of the Dirac operator exist:

$$
\mathcal{M}=\left\{y \in \mathbf{R}^{D} \mid E_{0}(y)=0\right\} .
$$

This definition of the classical space may look similar to that using the Hamiltonian (2.7). A crucial difference is that in the method using the Dirac operator, we do not need to take the large- $N$ limit to define the classical space $\mathcal{M}$. The Dirac operator allows exact zero modes even for finite $N$, and the geometry is defined for a fixed finite $N$.

There are two different interpretations of this construction in the context of the string theory. One is based on the probe picture of D0-brane action [18]. Suppose $N$ D0-branes form a bound state such as fuzzy sphere and behave as higher dimensional D-brane. Let $X^{\mu}$ be the matrix configuration (the bosonic fields) of these D0-branes. In addition, we consider another probe D0-brane at $y^{\mu}$. Then, the Dirac operator (2.9) appears in the fermionic kinetic term of the open string modes connecting the bounded D0-branes and the probe brane. $E_{0}$ measures the lowest energy of the open string, which is in general proportional to the length of the open string. Thus, at the position where Dirac zero modes exist, the probe brane hits the D0-branes, and hence $\mathcal{M}$ defined by (2.12) gives the geometry of the D0-branes seen by the probe brane.

The second interpretation is provided by flat non-BPS D-brane systems in superstring theory [22,23] (see also $[24,25])$. The theory on the non-BPS D-branes generally contains the tachyon field $T(y)$, and the potential term of $T(y)$ in the low energy action is proportional to the exponential factor $e^{-T(y)^{2}}$. The theory possesses a classical solution, which represents tachyon condensations. The solution takes the form $T(y)=u \not D(y)$, where $X^{\mu}$ in (2.9) can be arbitrary constant Hermitian matrices. In order for this to be a solution of the equation of motion, the parameter $u$ has to be sent to infinity. Then, since the potential energy is proportional to $e^{-u^{2} \not(y)^{2}}$ with $u \rightarrow \infty$, only zero modes of the Dirac operator survive. In particular, this is possible only when $y \in \mathcal{M}$, where $\mathcal{M}$ is defined by (2.12). Thus, this solution corresponds to a situation that the original non-BPS branes with the world volume coordinates $y^{\mu}$ becomes another configurations of D-branes which has the shape of $\mathcal{M}$. From the analysis of the boundary string field theory, the latter D-branes are found to be stable BPS D-branes. Thus, in this context, $\mathcal{M}$ given by (2.12) corresponds to the shape of the BPS D-branes produced after the tachyon condensation.

The square of the Dirac operator is calculated as

$$
D^{2}(y)=\mathbb{1}_{2^{[D / 2]}} \otimes\left(X^{\mu}-y^{\mu} I_{N}\right)^{2}+\frac{1}{4}\left[\Gamma^{\mu}, \Gamma^{\nu}\right] \otimes\left[X^{\mu}, X^{\nu}\right] .
$$

Note that the first term on the right-hand side is proportional to the Hamiltonian. For the commuting matrices in the large- $N$ limit, we have

$$
D^{2}(y) \simeq 2 \mathbb{1}_{2^{[D / 2]}} \otimes H(y)
$$

in the large- $N$ limit. Thus, the Dirac operator asymptotically coincides with the Hamiltonian in the large- $N$ limit. One may think that the relation (2.14) between the Hamiltonian and the Dirac operator in the large- $N$ limit also implies the equivalence of the classical spaces defined by the two methods. However, rigorously speaking, there are some cases in which the classical spaces do not coincide with each other. Let us denote by $\mathcal{M}_{H}$ and $\mathcal{M}_{\not \supset}$ the classical spaces defined by the Hamiltonian and the Dirac operator in the large- $N$ limit, respectively. For $y^{\mu} \in \mathcal{M}_{\not}$, there exists a zero mode of the Dirac operator as $\not D(y)|0, y\rangle=0$. Then we have

$0=\left\langle 0, y\left|\not D^{2}(y)\right| 0, y\right\rangle \simeq 2\left\langle 0, y\left|\mathbb{1}_{2^{[D / 2]}} \otimes H(y)\right| 0, y\right\rangle$. 
Thus, there also exists a zero mode of the Hamiltonian at the same point $y^{\mu}$ in the large- $N$ limit. This means that $\mathcal{M}_{\varnothing} \subset \mathcal{M}_{H}$. However, the inverse statement may not be true in general. For $y^{\mu} \in \mathcal{M}_{H}$, one can only say that $\not D$ has an approximate zero mode in the large- $N$ limit, namely, $\not$ may not have an exact zero mode at that point. Thus, if there exists a point at which any eigenvalues of $\not D$ are nonzero but some of them are very small as $\mathcal{O}(1 / N)$, such point will be included in $\mathcal{M}_{H}$ but not in $\mathcal{M}_{\not \supset}$. Hence, in general, we have only the relation $\mathcal{M}_{\varnothing} \subset \mathcal{M}_{H}$.

The method using the Dirac operator has an advantage that the geometry can be rigidly defined at finite $N$ and hence mathematically rigorous treatment is possible at finite $N$. However, depending on a context, one may be interested in the geometry which emerges only in the large$N$ limit. The method using the Hamiltonian has an advantage that, without introducing the vector space of spinors, one can pick up not only the points in $\mathcal{M}_{\not \supset}$ but also approximately emergent points.

In the both pictures, each zero eigenstate describes a single D-brane. If there are some degenerate zero eigenstates of the Dirac operator, they corresponds to multiple coincident D-branes.

\section{INFORMATION METRIC AND BERRY CONNECTION}

In this section, we give definitions of the information metric and Berry connection on $\mathcal{M}$. In this section, we use the Dirac operator method, but the same arguments apply to the Hamiltonian method as well. See [21] for the case of the Hamiltonian method.

\section{A. Information metric}

Suppose that the Dirac operator has $k$ degenerate zero modes and the zero eigenstates are labeled as $|0, a, y\rangle$ $(a=1,2, \ldots, k)$. From these eigenstates, we first define a density matrix,

$$
\rho(y)=\frac{1}{k} \sum_{a=1}^{k}|0, a, y\rangle\langle 0, a, y|,
$$

which is proportional to the projection operator onto the $k$-dimensional vector space spanned by $|0, a, y\rangle$. Note that $\rho(y)$ is the unique density matrix made of the zero eigenstates and invariant under the $U(k)$ rotational transformation of the zero eigenstates,

$|0, a, y\rangle \mapsto \sum_{b=1}^{k}|0, b, y\rangle V_{b a}(y), \quad V(y) \in U(k)$.

We consider the case that $\mathcal{M}$ defined in (2.12) is a smooth simply connected compact manifold corresponding to extended D-branes. ${ }^{4}$ On a vicinity of this manifold, $|0, a, y\rangle$ are differentiable. (Note that $|0, a, y+\epsilon\rangle=$ $|0, a, y\rangle+\epsilon^{\mu} \partial_{\mu}|0, a, y\rangle+\cdots$. The derivative terms are explicitly given by the formula of the perturbation theory under $\not D(y+\epsilon)=\not D(y)-\Gamma^{\mu} \epsilon_{\mu}$. This perturbation should be smooth at least when $\epsilon^{\mu}$ is much smaller than the spectral gap of the Dirac operator.) Then, $\rho$ defined by (3.1) gives a smooth map from $\mathcal{M}$ to the space of the density matrices. Furthermore, we can show that $\rho$ and its differential $d \rho$ are injective mappings. See Appendix A for our proof. Then, $\rho$ gives an embedding of $\mathcal{M}$ into the space $\mathcal{D}$ of all density matrices,

$$
\rho: \mathcal{M} \rightarrow \mathcal{D}
$$

The space $\mathcal{D}$ of the density matrices forms a convex cone and one can define a metric structure on this space. In fact, the information (Bures) metric provides a natural metric on $\mathcal{D}$, defined by

$$
d s^{2}=\frac{1}{2} \operatorname{Tr}(d \rho G), \quad d \rho=\rho G+G \rho .
$$

Here, the trace is taken over the vector space associated with the density matrices and $G$ is defined from $\rho$ by the second equation of (3.4). For pure states, the information metric is equivalent to the Fubini-Study metric on the complex projective space given by a set of normalized complex vectors.

The embedding (3.3) then defines the pullback of the information metric. This pullback provides a metric structure for $\mathcal{M}$. For $\rho$ given by (3.1), differentiating the relation $\rho^{2}=\rho / k$, one finds that

$$
G=k d \rho
$$

The pullback can then be explicitly written as

$$
d s^{2}=\frac{1}{k}\left(\sum_{a=1}^{k} \| d|0, a, y\rangle \|^{2}-\sum_{a, b=1}^{k}|\langle 0, a, y|d| 0, b, y\rangle|^{2}\right) .
$$

Here $\|\cdot\|$ is the vector norm and $d|0, a, y\rangle$ is understood as

$$
|0, a, d y\rangle=\frac{\partial}{\partial \sigma^{\alpha}}|0, a, y\rangle d \sigma^{\alpha},
$$

where $\left\{\sigma^{\alpha}\right\}$ is arbitrary local coordinate on $\mathcal{M}$. The local coordinate should be chosen such that $\left\{\sigma^{\alpha}\right\}$ parametrizes the zeros of $\not(y)$ as $E_{0}(y(\sigma))=0$.

\footnotetext{
${ }^{4}$ In general, $\mathcal{M}$ contains some disconnected components. The following arguments can be easily extended to such general cases.
} 


\section{B. Berry connection}

We can consider a gauge connection on $\mathcal{M}$ associated with the local $U(k)$ rotation (3.2) of the zero eigenstates $|0, a, y\rangle$. This gauge field corresponds to the (non-Abelian) Berry connection. The Berry connection is defined as the following one form on $\mathcal{M}$ :

$$
A_{a b}(\sigma)=-i\langle 0, a, y|d| 0, b, y\rangle,
$$

where $d|0, b, y\rangle$ is defined in (3.7). It is easy to see that (3.8) transforms as a non-Abelian gauge field under the transformation (3.2) as

$$
A \rightarrow V^{\dagger} A V-i V^{\dagger} d V
$$

For well-known fuzzy spaces such as fuzzy $S^{2}$ and $S^{4}$, this gauge field takes topologically nontrivial configurations such as monopoles and instantons. We demonstrate this calculation in the following sections.

Let us comment on the setup considered in [21], in which matrices $\left\{X^{\mu}\right\}$ behave as

$$
\left[X^{\mu}, X^{\nu}\right]=\frac{i}{c_{N}} W^{\mu \nu}(X)+\cdots .
$$

Here, $c_{N}$ is an $N$-dependent constant which goes to infinity in the large- $N$ limit, and $\cdots$ represents higher order terms in $1 / c_{N} . W^{\mu \nu}(X)$ in (3.10) is antisymmetric in the indices $\mu, \nu$ and is a polynomial in $X^{\mu}$ with convergent degree and coefficients in the large- $N$ limit. For the matrices satisfying (3.10), it was shown in [21] that the curvature 2-form of the Berry connection gives a symplectic form in the large- $N$ limit. Namely, the curvature 2-form is closed and nondegenerate. The information metric was also shown to be the compatible Kähler metric for the symplectic form.

In the setup with D-branes studied in $[18,22,23]$, the Berry connection is understood as the gauge field on the D-branes, as first noted in $[24,26]$. For Kähler manifolds, the information metric is the compatible world volume metric on the D-branes.

\section{EXAMPLES}

In this section, we consider fuzzy $S^{2}$ and $S^{4}$ as examples. Through explicit calculations, we demonstrate that the information metric for these spaces are given by the ordinary round metric, while the Berry connections are given by the configurations of the $\mathrm{Wu}$-Yang monopole and Yang monopole for fuzzy $S^{2}$ and $S^{4}$, respectively.

\section{A. Fuzzy $S^{2}$}

\section{Definition of fuzzy $S^{2}$}

In the standard description of the fuzzy $S^{2}$, one uses three Hermitian matrices, which correspond to the quantized embedding functions into $\mathbf{R}^{3}$. The three matrices are given as

$$
X^{i}=R L^{i}, \quad R=\frac{2}{\sqrt{N^{2}-1}} .
$$

Here, $L^{i}$ are the $S U(2)$ generators in the spin- $J$ irreducible representation, where $J$ is related to the matrix size $N$ by $N=2 J+1$. The normalization factor $R$ is chosen so that the fuzzy sphere has a unit radius as

$$
\sum_{i=1}^{3}\left(X^{i}\right)^{2}=1 .
$$

These matrices satisfy the commutation relations ${ }^{5}$

$$
\left[X^{i}, X^{j}\right]=\frac{2 i \epsilon^{k i j}}{\sqrt{N^{2}-1}} X^{k} .
$$

For later convenience, we introduce the standard basis $|J, m\rangle$ of the representation space of $L^{i}$. They satisfy

$$
\begin{aligned}
L^{3}|J, m\rangle & =m|J, m\rangle, \\
L^{ \pm}|J, m\rangle & =\sqrt{(J \mp m)(J \pm m+1)}|J, m \pm 1\rangle,
\end{aligned}
$$

where $L^{ \pm}=L^{1} \pm i L^{2}$. For $J=1 / 2$, we also use the shorthand notation,

$$
|1 / 2, \pm\rangle:=|1 / 2, \pm 1 / 2\rangle .
$$

\section{Classical space for fuzzy $S^{2}$}

The Dirac operator for fuzzy $S^{2}$ is given by a $2 N \times 2 N$ Hermitian matrix,

$$
\not(y)=\sigma^{i} \otimes\left(R L^{i}-y^{i}\right) .
$$

Here $\sigma^{i}$ are the Pauli matrices. The spectrum of (4.6) is derived in the Appendix B (See also [19,20,22]). There are three types of the eigenstates,

$$
\begin{aligned}
\left|\psi_{m}^{( \pm)}\right\rangle= & \left(U_{2} \otimes U_{N}\right)\left(a_{m}^{( \pm)}|1 / 2,+\rangle \otimes|J, m\rangle+b_{m}^{( \pm)}|1 / 2,-\rangle\right. \\
& \otimes|J, m+1\rangle), \\
\left|\psi_{J}\right\rangle= & \left(U_{2} \otimes U_{N}\right)|1 / 2,+\rangle \otimes|J, J\rangle, \\
\left|\psi_{\bullet}\right\rangle= & \left(U_{2} \otimes U_{N}\right)|1 / 2,-\rangle \otimes|J,-J\rangle,
\end{aligned}
$$

where $m=-J,-J+1, \ldots, J-2, J-1$, and the corresponding eigenvalues are given by

\footnotetext{
${ }^{5}$ Note that this commutation relation is of the form of (3.10). Thus, the argument in [21] can be applied.
} 


$$
\begin{aligned}
\lambda_{m}^{( \pm)}(y) & =-\frac{R}{2} \pm \frac{1}{2} \sqrt{R^{2}+4\left\{|y|^{2}-R(2 m+1)|y|+R^{2} J(J+1)\right\}} \\
\lambda_{J}(y) & =R J-|y|, \\
\lambda \cdot(y) & =R J+|y|,
\end{aligned}
$$

respectively, where $|y|=\sqrt{\sum_{i} y_{i}^{2}}$. In (4.7), $a_{m}^{( \pm)}, b_{m}^{( \pm)}$are real coefficients satisfying

$$
a_{m}^{( \pm)}=-\frac{R \sqrt{(J-m)(J+m+1)}}{R m-|y|-\lambda_{m}^{( \pm)}} b_{m}^{( \pm)}
$$

as well as the normalization condition

$$
a^{( \pm) 2}+b_{m}^{( \pm) 2}=1 .
$$

The unitary matrices $U_{2}$ and $U_{N}$ in (4.7) are defined in Appendix C 1 .

Note that $\lambda_{\bullet}(y)$ is strictly positive and $\lambda_{m}^{( \pm)}(y)$ cannot be zero for $m=-J,-J+1, \ldots, J-1$. Thus, only $\left|\psi_{J}\right\rangle$ can be the zero mode of the Dirac operator. The classical space is then defined as a set of $y \in \mathbf{R}^{3}$ on which the zero mode exists:

$$
\mathcal{M}=\left\{y^{i} \in \mathbf{R}^{3}|| y \mid=R J\right\} .
$$

Obviously, the classical space is given by a sphere with the radius $R J$ embedded in $\mathbf{R}^{3}$. We can also apply the method using the Hamiltonian. This is shown in Appendix D 1.

\section{Information metric and Berry connection for fuzzy $S^{2}$}

We parametrize the classical space (4.11) by

$$
\begin{aligned}
& y^{1}=R J \sin \theta \cos \phi, \\
& y^{2}=R J \sin \theta \sin \phi, \\
& y^{3}=R J \cos \theta .
\end{aligned}
$$

We also introduce the stereographic coordinate $(z, z)$ on the classical space by

$$
z=e^{i \phi} \tan \frac{\theta}{2}
$$

We first compute the information metric (3.6) for the zero eigenstate $\left|\psi_{J}\right\rangle$ for the fuzzy $S^{2}$. The expression (C8) for the unitary matrix $U$ is very useful in computing the differential of the zero mode $\left|\psi_{J}\right\rangle$. In the stereographic coordinate, the differential of $U_{N}(y)|J, J\rangle$ is given by

$$
\begin{aligned}
d U_{N}(y)|J, J\rangle= & \frac{J(\bar{z} d z-z d \bar{z})}{1+|z|^{2}} U_{N}(y)|J, J\rangle \\
& +\frac{\sqrt{2 J} d z}{1+|z|^{2}} U_{N}(y)|J, J-1\rangle .
\end{aligned}
$$

By using this, we can easily compute (3.6). The result is given by

$d s^{2}=\| d\left|\psi_{J}\right\rangle||^{2}-\left|\left\langle\psi_{J}|d| \psi_{J}\right\rangle\right|^{2}=N \frac{d z d \bar{z}}{\left(1+|z|^{2}\right)^{2}}$.

This is nothing but a round Kähler metric for $S^{2}$. The overall factor also picks up information of the density of D0-branes, which is an intrinsic data of the matrices $X^{\mu}$.

By using (4.14), we can also compute the Berry connection (3.8) for fuzzy $S^{2}$. The result is given by

$$
A=-i\left\langle\psi_{J}|d| \psi_{J}\right\rangle=-i \frac{N \bar{z} d z-z d \bar{z}}{2} .|z|^{2} .
$$

This is just the Dirac monopole configuration. The field strength is

$$
F=d A=i N \frac{d z \wedge d \bar{z}}{\left(1+|z|^{2}\right)^{2}}
$$

The monopole flux (or equivalently the first Chern class) coincides with the matrix size $N$ :

$$
\frac{1}{2 \pi} \int F=N
$$

\section{B. Fuzzy $S^{4}$}

\section{Definition of fuzzy $S^{4}$}

Let us first introduce the following orthonormal vectors ${ }^{6}$ :

$$
\begin{aligned}
& \left|\eta_{1}\right\rangle=\left(\begin{array}{l}
1 \\
0 \\
0 \\
0
\end{array}\right), \quad\left|\eta_{2}\right\rangle=\left(\begin{array}{l}
0 \\
1 \\
0 \\
0
\end{array}\right), \\
& \left|\eta_{3}\right\rangle=\left(\begin{array}{l}
0 \\
0 \\
1 \\
0
\end{array}\right), \quad\left|\eta_{4}\right\rangle=\left(\begin{array}{l}
0 \\
0 \\
0 \\
1
\end{array}\right) .
\end{aligned}
$$

We denote by $\mathcal{H}_{n}$ the Hilbert space spanned by all $n$-fold totally symmetric tensor products of $\left|\eta_{i}\right\rangle(i=1,2,3,4)$. We denote by $N$ the dimension of this space:

$$
N=\operatorname{dim} \mathcal{H}_{n}=\left(\begin{array}{c}
n+3 \\
3
\end{array}\right)=\frac{(n+1)(n+2)(n+3)}{6} .
$$

\footnotetext{
${ }^{6}$ See also $[9,27]$ for the calculation in this subsection.
} 
We also denote by $\mathcal{H}_{n}^{+}$the subspace of $\mathcal{H}_{n}$ spanned only by all symmetric tensor products of $\left|\eta_{1}\right\rangle$ and $\left|\eta_{2}\right\rangle$. The dimension of this subspace is

$$
\operatorname{dim} \mathcal{H}_{n}^{+}=\left(\begin{array}{c}
n+1 \\
1
\end{array}\right)=n+1 .
$$

We also introduce the five dimensional gamma matrices $\Gamma^{A}$, satisfying $\left\{\Gamma^{A}, \Gamma^{B}\right\}=2 \delta^{A B} \mathbb{1}_{4}$. In the following, we use the following representation of $\Gamma_{A}$ :

$$
\begin{gathered}
\Gamma_{i}=\sigma_{2} \otimes \sigma_{i}=\left(\begin{array}{cc}
0 & -i \sigma_{i} \\
i \sigma_{i} & 0
\end{array}\right) \quad i \in\{1,2,3\}, \\
\Gamma_{4}=\sigma_{1} \otimes \mathbb{1}_{2}=\left(\begin{array}{cc}
0 & \mathbb{1}_{2} \\
\mathbb{1}_{2} & 0
\end{array}\right), \\
\Gamma_{5}=\sigma_{3} \otimes \mathbb{1}_{2}=\left(\begin{array}{cc}
\mathbb{1}_{2} & 0 \\
0 & -\mathbb{1}_{2}
\end{array}\right),
\end{gathered}
$$

where $\sigma_{i}$ are the Pauli matrices. Note that $\left|\eta_{i}\right\rangle$ are eigenvectors of $\Gamma_{5}$ with $\Gamma_{5}\left|\eta_{1,2}\right\rangle=\left|\eta_{1,2}\right\rangle$ and $\Gamma_{5}\left|\eta_{3,4}\right\rangle=-\left|\eta_{3,4}\right\rangle$.

The vector space $\mathcal{H}_{n}$ gives the $N$-dimensional irreducible representation space of the $S O(5)$ Lie group. The $S O(5)$ generators $\Sigma_{A B}(A, B=1,2, \ldots, 5)$ are represented on $\mathcal{H}_{n}$ as

$$
\begin{aligned}
D_{\mathcal{H}_{n}}\left(\Sigma_{A B}\right)= & \frac{1}{2}\left(\Gamma_{A B} \otimes \mathbb{1}_{4} \otimes \cdots \otimes \mathbb{1}_{4}+\mathbb{1}_{4} \otimes \Gamma_{A B}\right. \\
& \left.\otimes \cdots \otimes \mathbb{1}_{4}+\cdots+\mathbb{1}_{4} \otimes \cdots \otimes \mathbb{1}_{4} \otimes \Gamma_{A B}\right) .
\end{aligned}
$$

The fuzzy $S^{4}$ is defined by the configuration of the five matrices on $\mathcal{H}_{n}$,

$$
X_{A}=R G_{A}^{(n)}, \quad R=\frac{1}{n},
$$

for $A=1,2, \ldots, 5$. Here, $G_{A}^{(n)}$ are $N \times N$ matrices acting on $\mathcal{H}_{n}$ and are given by the $n$-fold symmetric tensor products of the five-dimensional Euclidean gamma matrices $\Gamma_{A}$ :

$$
\begin{aligned}
G_{A}^{(n)}= & \Gamma_{A} \otimes \mathbb{1}_{4} \otimes \cdots \otimes \mathbb{1}_{4}+\mathbb{1}_{4} \otimes \Gamma_{A} \otimes \cdots \otimes \mathbb{1}_{4}+\cdots \\
& +\mathbb{1}_{4} \otimes \cdots \otimes \mathbb{1}_{4} \otimes \Gamma_{A} .
\end{aligned}
$$

We emphasize that though $G_{A}^{(n)}$ are represented as $4^{n} \times 4^{n}$ matrices, the Hilbert space is now restricted to $\mathcal{H}_{n}$ with dimension (4.20). As we prove in Appendix E, the matrices $G_{A}^{(n)}$ satisfy the relation,

$$
\sum_{A}\left(G_{A}^{(n)}\right)^{2}=n(n+4) \mathbb{1}_{\mathcal{H}_{n}} .
$$

The normalization factor $R$ is chosen so that $X_{A}$ gives a unit sphere in the large- $N$ limit:

$$
\sum_{A} X_{A}^{2}=\mathbb{1}_{\mathcal{H}_{n}}+\mathcal{O}(1 / n)
$$

Below, we will see that the classical spaces defined by the Dirac operator and Hamiltonian indeed become the unit sphere in the large- $N$ limit.

\section{Classical space for fuzzy $S^{4}$}

Here, we compute the classical space of fuzzy $S^{4}$ by using the Dirac operator method (See also [20]). See Appendix D 2 for the derivation using the Hamiltonian method.

For the configuration (4.24) of fuzzy $S^{4}$, the Dirac operator is given by

$$
\not D(y)=\Gamma^{A} \otimes\left(R G_{A}^{(n)}-y_{A}\right) .
$$

This is a $4 N \times 4 N$ Hermitian matrix acting on $C^{4} \otimes \mathcal{H}_{n}$. In [20], it is shown that this Dirac operator has $n+2$ degenerate zero modes. In the following, we present these states based on a symmetry argument. We parametrize $y_{A}$ as $y_{A}=|y| x_{A}$, where $|y|=\sqrt{\sum_{A} y_{A}^{2}}$ and $x_{A}$ is the unit vector (C9) parametrized with the polar coordinates. We consider a similarity transformation of $\boldsymbol{D}(y)$ with the unitary matrix defined in (C17). Because of the relations (C11) and (C18), the Dirac operator transforms into

$$
U^{\dagger \otimes(n+1)} \not(y) U^{\otimes(n+1)}=\sum_{a=1}^{4} \Gamma^{a} \otimes R G_{a}^{(n)}+\Gamma^{5} \otimes\left(R G_{5}^{(n)}-|y|\right) .
$$

By using (E3) and $\Gamma^{5}\left|\eta_{1}\right\rangle=\left|\eta_{1}\right\rangle$, we can see that the $(n+1)$-fold tensor product of $\left|\eta_{1}\right\rangle$ gives an eigenstate of (4.29) with the eigenvalue $n R-|y|$. Thus, for $|y|=n R$, $U^{\otimes(n+1)}\left|\eta_{1}\right\rangle^{\otimes(n+1)}$ gives a zero mode of the Dirac operator. Note that the vector $\left|\eta_{1}\right\rangle^{\otimes(n+1)}$ is an element of $\mathcal{H}_{n+1} \subset C^{4} \otimes \mathcal{H}_{n}$. Hence, we can consider the action of $S O(5)$ generators $D_{\mathcal{H}_{n+1}}\left(\Sigma_{A B}\right)$ onto this vector. We notice that (4.29) commutes with the $S O(4)$ generators $D_{\mathcal{H}_{n+1}}\left(\Sigma_{a b}\right)$ with $a, b=1,2,3,4$, since all the $S O(4)$ vector indices are contracted in (4.29). Thus, any states given by acting these generators on $\left|\eta_{1}\right\rangle^{\otimes(n+1)}$ also give zero eigenstates of (4.29). In order to write down these states, we utilize the decomposition of the $S O(4)$ generators into the generators of $S U(2) \times S U(2)$ :

$$
\begin{aligned}
J_{1} & =-\frac{i}{2}\left(\Sigma_{41}+\Sigma_{23}\right), \quad J_{2}=-\frac{i}{2}\left(\Sigma_{42}+\Sigma_{31}\right), \\
J_{3} & =-\frac{i}{2}\left(\Sigma_{43}+\Sigma_{12}\right), \\
\tilde{J}_{1} & =\frac{i}{2}\left(\Sigma_{41}-\Sigma_{23}\right), \quad \tilde{J}_{2}=\frac{i}{2}\left(\Sigma_{42}-\Sigma_{31}\right), \\
\tilde{J}_{3} & =\frac{i}{2}\left(\Sigma_{43}-\Sigma_{12}\right) .
\end{aligned}
$$

They satisfy 


$$
\left[J_{i}, J_{j}\right]=i \epsilon_{i j k} J_{k},\left[\tilde{J}_{i}, \tilde{J}_{j}\right]=i \epsilon_{i j k} \tilde{J}_{k}, \quad\left[J_{i}, \tilde{J}_{j}\right]=0 .
$$

It is easy to see that $D_{\mathcal{H}_{n+1}}\left(\tilde{J}_{i}\right)$ are vanishing on $\left|\eta_{1}\right\rangle^{\otimes(n+1)}$, while $D_{\mathcal{H}_{n+1}}\left(J_{i}\right)$ act as

$$
\begin{aligned}
D_{\mathcal{H}_{n+1}}\left(J_{3}\right)\left|\eta_{1}\right\rangle^{\otimes(n+1)} & =\frac{n+1}{2}\left|\eta_{1}\right\rangle^{\otimes(n+1)}, \\
D_{\mathcal{H}_{n+1}}\left(J_{i}\right)^{2}\left|\eta_{1}\right\rangle^{\otimes(n+1)} & =\frac{(n+1)(n+3)}{4}\left|\eta_{1}\right\rangle^{\otimes(n+1)} .
\end{aligned}
$$

Hence, the state $\left|\eta_{1}\right\rangle^{\otimes(n+1)}$ is the highest weight state under the one of the $S U(2)$ symmetries. We use the notation $J=$ $\frac{n+1}{2}$ for the spin of this state and label the highest state as

$$
\left|\eta_{1}\right\rangle^{\otimes(n+1)}=|J, J\rangle .
$$

By acting $D_{\mathcal{H}_{n+1}}\left(J_{-}\right)$on this state, we can obtain the other zero eigenstates of (4.29). By multiplying $U^{\otimes(n+1)}$ on these states, we finally obtain the $n+2$ degenerate zero eigenstates of $\not{D}(y)$ as

$$
\begin{aligned}
U^{\otimes(n+1)}|J, m\rangle= & \sqrt{\frac{(J+m) !}{(2 J) !(J-m) !}} U^{\otimes(n+1)} \\
& \times D_{\mathcal{H}_{n+1}}\left(J_{-}\right)^{J-m}\left|\eta_{1}\right\rangle^{\otimes(n+1)},
\end{aligned}
$$

where $m=-J,-J+1, \ldots, J$. These states have the common eigenvalue $R n-|y|$ for the Dirac operator.

The classical space is given by the loci of zeros of the Dirac operator as

$$
\mathcal{M}=\left\{y \in \mathbf{R}^{5}|| y \mid=n R\right\} .
$$

This is indeed $S^{4}$ with radius $n R=1$. Note that this radius differs from the naive expectation (4.27) by $1 / n$ corrections.

\section{Information metric and Berry connection for fuzzy $S^{4}$}

We introduce the spherical coordinate for (4.35) by parametrizing $y^{A}$ as

$$
y^{A}=R n x^{A},
$$

where $x^{A}$ is defined in (C9). The information metric for the fuzzy $S^{4}$ is given by

$$
\begin{aligned}
d^{2} s= & \frac{1}{n+2}\left(\sum_{m=-J}^{J} \| d U^{\otimes(n+1)}|J, m\rangle \|^{2}\right. \\
& \left.-\sum_{m, m^{\prime}=-J}^{J}\left|\left\langle J, m\left|U^{\dagger \otimes(n+1)} d U^{\otimes(n+1)}\right| J, m^{\prime}\right\rangle\right|^{2}\right) .
\end{aligned}
$$

To evaluate this metric, let us introduce the chiral projection operators,

$$
P_{ \pm}=\frac{1}{2}\left(\mathbb{1}_{4} \pm \Gamma_{5}\right)
$$

Note that the states $|J, m\rangle$ have the positive chirality,

$$
P_{ \pm}^{\otimes(n+1)}|J, m\rangle=|J, m\rangle .
$$

We notice that the second term in (4.37) can be written as

$$
\begin{aligned}
& -\frac{1}{n+2} \sum_{m, m^{\prime}=-J}^{J}\left\langle J, m\left|U^{\dagger \otimes(n+1)} d U^{\otimes(n+1)}\right| J, m^{\prime}\right\rangle \\
& \quad \times\left\langle J, m^{\prime}\left|d U^{\dagger \otimes(n+1)} U^{\otimes(n+1)}\right| J, m\right\rangle,
\end{aligned}
$$

and $U^{\dagger} d U$ takes values in the $S O(5)$ Lie algebra. Since the both sides of $U^{\dagger} d U$ are projected onto the positive chirality states in (4.40), only terms with $S O(4)$ generators survive. Furthermore, if one decomposes $S O(4)$ to $S U(2) \times S U(2)$ as in (4.30), $\tilde{J}_{i}$ vanish on $|J, m\rangle$. Thus, only the generators $J_{i}$ in $U^{\dagger} d U$ survive in computing (4.40). For the generators $J_{i}, \sum_{m^{\prime}}\left|J, m^{\prime}\right\rangle\left\langle J, m^{\prime}\right|$ behaves as the unit matrix. In other words, we have

$$
\sum_{m=-J}^{J}|J, m\rangle\langle J, m|=P_{+}^{\otimes(n+1)} .
$$

Hence, (4.40) is equivalent to

$$
\begin{aligned}
& -\frac{1}{n+2} \sum_{m=-J}^{J}\langle J, m| U^{\dagger \otimes(n+1)} d U^{\otimes(n+1)} P_{+}^{\otimes(n+1)} \\
& \quad \times d U^{\dagger \otimes(n+1)} U^{\otimes(n+1)}|J, m\rangle .
\end{aligned}
$$

Combining this with the first term in (4.37), we find that the information metric is written as

$$
\begin{aligned}
d s^{2} & =\frac{1}{n+2} \sum_{m=-J}^{J}\langle J, m|\mathcal{O}| J, m\rangle, \\
\mathcal{O} & =P_{+} d U^{\dagger} U P_{-} U^{\dagger} d U P_{+} \otimes \mathbb{1}_{4}^{\otimes n}+\cdots,
\end{aligned}
$$

where $\cdots$ stands for the $(n+1)$-fold symmetrization of the first term. From (C7), we can explicitly compute $U^{\dagger} d U$ as

$$
\begin{aligned}
U^{\dagger} d U= & \frac{1}{2} d \theta \Gamma_{45}+\frac{1}{2} d \phi\left(\cos \theta \Gamma_{34}+\sin \theta \Gamma_{35}\right) \\
& +\frac{1}{2} d \psi\left\{\cos \phi \Gamma_{23}+\sin \phi\left(\cos \theta \Gamma_{24}+\sin \theta \Gamma_{25}\right)\right\} \\
& +\frac{1}{2} d \chi\left[\cos \psi \Gamma_{12}+\sin \psi\left\{\cos \phi \Gamma_{13}\right.\right. \\
& \left.\left.+\sin \phi\left(\cos \theta \Gamma_{14}+\sin \theta \Gamma_{15}\right)\right\}\right]
\end{aligned}
$$


This is decomposed under the chiral projection as

$$
\begin{aligned}
P_{+} U^{\dagger} d U P_{+}= & P_{+}\left\{\frac{d \phi}{2} \cos \theta \Gamma_{34}\right. \\
& +\frac{d \psi}{2}\left(\cos \phi \Gamma_{23}+\sin \phi \cos \theta \Gamma_{24}\right) \\
& +\frac{d \chi}{2}\left(\cos \psi \Gamma_{12}+\sin \psi \cos \phi \Gamma_{13}\right. \\
& \left.\left.+\sin \psi \sin \phi \cos \theta \Gamma_{14}\right)\right\} P_{+}, \\
P_{-} U^{\dagger} d U P_{+}= & P_{-}\left\{\frac{d \theta}{2} \Gamma_{45}+\frac{d \phi}{2} \sin \theta \Gamma_{35}\right. \\
& +\frac{d \psi}{2} \sin \phi \sin \theta \Gamma_{25} \\
& \left.+\frac{d \chi}{2} \sin \psi \sin \phi \sin \theta \Gamma_{15}\right\} P_{+} .
\end{aligned}
$$

By substituting this into (4.43), finally we find that

$$
\begin{aligned}
d s^{2}= & \frac{n+1}{4}\left(d \theta^{2}+\sin ^{2} \theta d \phi^{2}+\sin ^{2} \theta \sin ^{2} \phi d \psi^{2}\right. \\
& \left.+\sin ^{2} \theta \sin ^{2} \phi \sin ^{2} \psi d \chi^{2}\right) .
\end{aligned}
$$

This is the standard round metric for $S^{4}$.

Next, we calculate the Berry connection for fuzzy $S^{4}$, which is defined by

$$
A_{m m^{\prime}}=-i\left\langle J, m\left|U^{\dagger \otimes(n+1)} d U^{\otimes(n+1)}\right| J, m^{\prime}\right\rangle .
$$

By using (4.30) and (4.45), we find that the Berry connection is given by

$$
\begin{aligned}
A_{m m^{\prime}}= & (\cos \phi d \psi-\sin \psi \sin \phi \cos \theta d \chi) D_{\mathcal{H}_{n+1}}\left(J_{1}\right)_{m m^{\prime}} \\
& -(\sin \phi \cos \theta d \psi+\sin \psi \cos \phi d \chi) D_{\mathcal{H}_{n+1}}\left(J_{2}\right)_{m m^{\prime}} \\
& -(\cos \theta d \phi-\cos \psi d \chi) D_{\mathcal{H}_{n+1}}\left(J_{3}\right)_{m m^{\prime}} .
\end{aligned}
$$

Let us also calculate the field strength. Introducing the matrix notation, $A:=\sum_{i=1}^{3} A_{i} D_{\mathcal{H}_{n+1}}\left(J_{i}\right)$, the field strength is given by $F=\sum_{i=1}^{3} F_{i} D_{\mathcal{H}_{n+1}}\left(J_{i}\right)$, where

$$
F^{a}=d A^{a}-\frac{1}{2} \epsilon^{a b c} A^{b} \wedge A^{c},
$$

Straightforward calculation gives

$F^{1}=-\sin \phi \sin ^{2} \theta d \phi \wedge d \psi+\sin \psi \sin \phi \sin \theta d \theta \wedge d \chi$,

$F^{2}=\sin \phi \sin \theta d \theta \wedge d \psi+\sin \psi \sin \phi \sin ^{2} \theta d \phi \wedge d \chi$,

$F^{3}=\sin \theta d \theta \wedge d \phi-\sin \psi \sin ^{2} \phi \sin ^{2} \theta d \psi \wedge d \chi$.

We can also show that this configuration is self-dual. Taking a square of the field strength, we obtain

$$
\begin{aligned}
F^{1} & \wedge F^{1}+F^{2} \wedge F^{2}+F^{3} \wedge F^{3} \\
& =6 \sin ^{3} \theta \sin ^{2} \phi \sin \psi(d \chi \wedge d \psi \wedge d \phi \wedge d \theta) .
\end{aligned}
$$

The right-hand side is just a volume form on $S^{4}$. This configuration is known as the $S U(2)$ Yang monopole on $S^{4}$. The instanton number (The second Chern class) is given by the matrix size:

$$
\frac{1}{8 \pi^{2}} \int \operatorname{Tr}_{\mathcal{H}_{n+1}}(F \wedge F)=N
$$

\section{BEREZIN-TOEPLITZ QUANTIZATION}

In this section, we show that the matrix configurations of fuzzy $S^{2}$ and fuzzy $S^{4}$ can be regarded as the images of the Berezin-Toeplitz quantization map.

\section{A. Review of Berezin-Toeplitz quantization}

We first give a brief review of the Berezin-Toeplitz quantization map on spin-C manifold. We consider a Euclidean compact spin-C manifold $\mathcal{M}$ with a Riemannian metric $g$ and a spinor bundle on $\mathcal{M}$. We assume that the gauge group is $U(k)$ and the spinors shall belong to the representation $\mathcal{R}$ of the gauge group. We define the Dirac operator as usual as

$$
\not D=\Gamma^{A} e_{A}^{\mu}\left(\partial_{\mu}+\frac{1}{4} \omega_{\mu B C} \Gamma^{B C}-i A_{\mu}\right)
$$

where $A, e$, and $\omega$ are the gauge connection, vielbein, and spin connection, respectively. By using the invariant measure defined from the metric $g$, we can define the inner product of sections. We denote this inner product as $\left(\psi, \psi^{\prime}\right)$.

Because of the index theorem, the kernel of the Dirac operator (5.1) forms a finite dimensional vector space. The dimension of this vector space is related to the Chern numbers of $A$ as well as the representation $\mathcal{R}$ of spinors. We denote this dimension by $N$. Let $\left\{\psi_{i} \mid i=1,2, \ldots, N\right\}$ be an orthonormal basis of $\operatorname{Ker} \not D$ satisfying $\left(\psi_{i}, \psi_{j}\right)=\delta_{i j}$. Multiplying a function $f \in C^{\infty}(\mathcal{M})$ on $\psi_{i}$ gives another spinor, which in general does not belong to $\operatorname{Ker} \not D$ and can be expanded in terms of the eigenfunctions of $\not D$ as

$$
f \psi_{i}=\sum_{j=1}^{N} \hat{f}_{i j} \psi_{j}+\cdots
$$

$\hat{f}_{i j}$ are constants (coefficients of $\psi_{j}$ in this expansion), and $\cdots$ represents the part which takes values in the orthogonal complement of Ker $\not D$. The coefficients $\hat{f}_{i j}$ can be extracted as

$$
\hat{f}_{i j}=\left(\psi_{j}, f \psi_{i}\right) .
$$


Since $\left\{\hat{f}_{i j}\right\}$ is just a constant $N \times N$ matrix, this construction can be seen as a mapping from a function $f$ to an $N \times N$ matrix. This is the Berezin-Toeplitz quantization map. The matrix $\hat{f}$ is called the Toeplitz operator of $f$.

\section{B. Berezin-Toeplitz quantization for fuzzy $S^{2}$}

Here, we will show that the matrix configuration (4.1) is equal to the Toeplitz operator of the standard embedding function $S^{2} \rightarrow \mathbf{R}^{3}$.

We first show that the zero eigenstate $\left|\psi_{J}\right\rangle$ in (4.7) also gives a zero eigenstate of the continuum Dirac operator (5.1). In order to fix the basis of the 2-component spinors, we make a local Lorentz transformation and consider

$$
\left.\left|\psi_{J}\right\rangle\right\rangle=U_{2}^{\dagger} \otimes 1_{N}\left|\psi_{J}\right\rangle=\left(\begin{array}{c}
U_{N}|J, J\rangle \\
0
\end{array}\right) .
$$

Note that $\left.\left|\psi_{J}\right\rangle\right\rangle$ contains only the positive chirality component $|1 / 2,+\rangle$, and this is written as the upper component in the last expression in (5.4). By using the vielbein and spin connection in Appendix F, we can write the covariant derivatives $\nabla_{a}=e_{a}^{\mu}\left(\partial_{\mu}+\frac{1}{4} \omega_{\mu b c} \sigma^{b c}\right)$ explicitly as

$$
\begin{aligned}
& \nabla_{+}=\frac{1+|z|^{2}}{r}\left(\partial_{z}+\frac{\bar{z}}{2\left(1+|z|^{2}\right)} \sigma^{3}\right), \\
& \nabla_{-}=\frac{1+|z|^{2}}{r}\left(\partial_{\bar{z}}-\frac{z}{2\left(1+|z|^{2}\right)} \sigma^{3}\right),
\end{aligned}
$$

where $r$ is the radius of the sphere. The actions of these operators on $\left.\left|\psi_{J}\right\rangle\right\rangle$ follow from (4.14) as

$$
\begin{aligned}
& \left.\left.\nabla_{+}\left|\psi_{J}\right\rangle\right\rangle=\frac{(J+1 / 2) \bar{z}}{r}\left|\psi_{J}\right\rangle\right\rangle+\frac{\sqrt{2 J}}{r}\left(\begin{array}{c}
U_{N}|J, J-1\rangle \\
0
\end{array}\right), \\
& \left.\left.\nabla_{-}\left|\psi_{J}\right\rangle\right\rangle=-\frac{(J+1 / 2) z}{r}\left|\psi_{J}\right\rangle\right\rangle .
\end{aligned}
$$

Note that the first terms in these expressions are just the Berry connections multiplied by the inverses of vielbein, $e_{a}^{\mu} A_{\mu}$. From (5.6), we find that $\left.\left|\psi_{J}\right\rangle\right\rangle$ satisfies

$$
\left.\left.\not D\left|\psi_{J}\right\rangle\right\rangle=\sigma^{a}\left(\nabla_{a}-i e_{a}^{\mu} A_{\mu}\right)\left|\psi_{J}\right\rangle\right\rangle=0 .
$$

Thus, $\left.\left|\psi_{J}\right\rangle\right\rangle$ is a zero eigenstate of $\not D$. There are $N$ independent components in $\left.\left|\psi_{J}\right\rangle\right\rangle$. Introducing the basis $|i\rangle(i=1,2, \ldots, N)$ of the $N$-dimensional vector space, we thus find $N$ zero modes of $\not D$ :

$$
\psi_{i}=\left(\begin{array}{c}
\left\langle i\left|U_{N}\right| J, J\right\rangle \\
0
\end{array}\right), \quad \not D \psi_{i}=0 .
$$

By using the information metric $g$, we can define the standard inner product for spinors. In the following calculations, we use the formulas,

$$
\int d \Omega_{2} \frac{|z|^{2 B}}{\left(1+|z|^{2}\right)^{A}}=4 \pi \frac{(A-B) ! B !}{(A+1) !}
$$

and

$U_{N}|J, J\rangle=\frac{1}{\left(1+|z|^{2}\right)^{J}} \sum_{r=-J}^{J} z^{J-r}\left(\begin{array}{c}2 J \\ J+r\end{array}\right)^{1 / 2}|J, r\rangle$,

where $d \Omega_{2}=\sin \theta d \theta \wedge d \phi=\frac{2 i d z \wedge d \bar{z}}{\left(1+|z|^{2}\right)^{2}}$ is the volume form of the unit sphere satisfying $\int d \Omega_{2}=4 \pi$. From these formulas, we can easily show that ${ }^{7}$

$$
\frac{N}{4 \pi} \int d \Omega_{2} U_{N}|J, J\rangle\langle J, J| U_{N}^{\dagger}=\mathbb{1}_{N} .
$$

This implies that $\psi_{i}$ are orthonormal under the inner product given by the information metric. From the index theorem, it also follows that the dimension of $\operatorname{Ker} \not D$ is equal to $N$. Thus, $\psi_{i}$ form an orthonormal basis of Ker $\not D$.

The Toeplitz operator for a function $f \in C^{\infty}\left(S^{2}\right)$ is given by

$\hat{f}_{i j}=\left(\psi_{j}, f \psi_{i}\right)=\frac{N}{4 \pi} \int d \Omega_{2}\left\langle i\left|U_{N}\right| J, J\right\rangle f\left\langle J, J\left|U_{N}^{\dagger}\right| j\right\rangle$.

The formula (5.11) also implies that the image of the unit constant function on $S^{2}$ is given by the identity matrix. Similarly, we can compute the images of the embedding functions $x^{i}(i=1,2,3)$ defined in $(\mathrm{C} 1)$. We find that they are mapped to

$$
\hat{x}^{i}=\frac{1}{J+1} L^{i},
$$

for $i=1,2,3$. This is just the matrix configuration of fuzzy $S^{2}$ up to the overall constant. Thus, the matrix configuration of the fuzzy $S^{2}$ can be regarded as the Toeplitz operator of the embedding function $S^{2} \rightarrow \mathbf{R}^{3}$.

The Toeplitz quantization map also induces mappings for derivatives and integrals on $S^{2}$. For example, the mapping rule for integrals on $S^{2}$ can be obtained by taking the trace of (5.12):

$$
\operatorname{Tr} \hat{f}=\frac{N}{4 \pi} \int d \Omega_{2} f
$$

Thus, integrals are mapped to traces. Similarly, we can derive the mapping rule for the Laplace operator on $S^{2}$ as

$$
(\hat{\Delta f})_{i j}=-\frac{1}{r^{2}}\left[L_{k},\left[L_{k}, \hat{f}\right]\right]_{i j}
$$

See Appendix G 1 for derivation.

\footnotetext{
${ }^{7}$ The Eq. (5.11) can also be obtained easily from the symmetry argument: The integration over $S^{2}$ only produces rotationally invariant tensors on $S^{2}$. From the structure of indices, the integration of $U_{N}|J, J\rangle\langle J, J| U_{N}^{\dagger}$ turns out to be proportional to the identity matrix. The proportionality constant is fixed by taking the trace.
} 


\section{Berezin-Toeplitz quantization for fuzzy $S^{4}$}

Next, we show that the matrix configuration (4.24) is equal to the Toeplitz operator of the standard embedding function $S^{4} \rightarrow \mathbf{R}^{5}$. We also obtain the mapping rule for the Laplacian on $S^{4}$.

We perform a local Lorentz transformation of the zero eigenstates (4.34) and consider

$$
\left.\left|\psi_{J m}\right\rangle\right\rangle=\mathbb{1}_{4} \otimes U^{\otimes n}|J, m\rangle .
$$

Recall that $|J, m\rangle$ is an element of $\mathcal{H}_{n+1} \subset C^{4} \times \mathcal{H}_{n}$ and can be written as a sum of tensor products of elements in $C^{4}$ and $\mathcal{H}_{n}$. This decomposition is given by

$|J, m\rangle=\sum_{s=-\frac{1}{2} \gamma=-J+\frac{1}{2}}^{\frac{1}{2}} \sum_{\frac{1}{2} s J-\frac{1}{2} \gamma}^{J-\frac{1}{2}}|1 / 2, s\rangle \otimes|J-1 / 2, \gamma\rangle$,

where $C_{a \alpha b \beta}^{c \gamma}$ is the Clebsh-Gordan coefficient of $S U(2)$. In terms of this expression, $\left.\left|\psi_{J m}\right\rangle\right\rangle$ can also be written as

$\left.\left|\psi_{J m}\right\rangle\right\rangle=\sum_{s=-\frac{1}{2}}^{\frac{1}{2}} \sum_{\gamma=-J+\frac{1}{2}}^{J-\frac{1}{2}} C_{\frac{1}{2} s J-\frac{1}{2} \gamma}^{J m}|1 / 2, s\rangle \otimes\left(U^{\otimes n}|J-1 / 2, \gamma\rangle\right)$.

Note that this vector has the positive chirality with respect to $\Gamma_{5}$ :

$$
\left.\left.\Gamma_{5} \otimes \mathbb{1}_{4}^{\otimes n}\left|\psi_{J m}\right\rangle\right\rangle=\left|\psi_{J m}\right\rangle\right\rangle .
$$

Below, we will show that $\left.\left|\psi_{J m}\right\rangle\right\rangle$ is a zero eigenvector of the differential Dirac operator (5.1). Here, the gauge field is given by the Berry connection (4.48) and the representation of the gauge group is the spin $J=\frac{n+1}{2}$ representation of $S U(2)$. The vielbein and the spin connection are given in Appendix F.

Let us first consider the action of the covariant derivative $\nabla_{a}=e_{a}^{\mu}\left(\partial_{\mu}+\frac{1}{4} \omega_{\mu b c} \Gamma^{b c}\right)$ without the gauge connection. By comparing (4.45) and (F7), we find that the spin connection has the following relation:

$$
\frac{1}{4} \sum_{a, b=1}^{4} \omega_{a b} \Gamma^{a b} P_{+}=P_{+} U^{\dagger} d U P_{+} .
$$

By using this relation, we obtain

$\left.\Gamma^{a} \nabla_{a}\left|\psi_{J m}\right\rangle\right\rangle=\left(\Gamma^{a} P_{+} \otimes U^{\otimes n}\right)\left(U^{\dagger} \partial_{a} U \otimes \mathbb{1}_{4}^{\otimes n}+\cdots\right)|J, m\rangle$,

where $\partial_{a}=e_{a}^{\mu} \partial_{\mu}$ and $\cdots$ stands for the symmetrization of the first term. In the symmetrization of $U^{\dagger} \partial_{a} U$, we insert $\mathbb{1}_{4}=P_{+}+P_{-}$in front of each $U^{\dagger} \partial_{a} U$. Then, the terms containing $P_{+}$in these insertions can be calculated as

$$
\begin{aligned}
& \left(\Gamma^{a} P_{+} \otimes\left(U P_{+}\right)^{\otimes n}\right)\left(U^{\dagger} \partial_{a} U \otimes \mathbb{1}_{4}^{\otimes n}+\cdots\right)|J, m\rangle \\
& =\sum_{m^{\prime}=-J}^{J}\left(\Gamma^{a} \otimes U^{\otimes n}\right)\left|J, m^{\prime}\right\rangle\left\langle J, m^{\prime}\left|\left(U^{\dagger} \partial_{a} U \otimes \mathbb{1}_{4}^{\otimes n}+\cdots\right)\right| J, m\right\rangle \\
& \left.=\sum_{m^{\prime}=-J}^{J}\left(\Gamma^{a} \otimes \mathbb{1}_{4}^{\otimes n}\right)\left|\psi_{J m^{\prime}}\right\rangle\right\rangle\left(i A_{a}\right)_{m^{\prime} m},
\end{aligned}
$$

where the second line follows from (4.41). Thus, this contribution gives the Berry connection. On the other hand, the terms containing $P_{-}$can be calculated as

$$
\begin{aligned}
& \left(\Gamma^{a} P_{+} \otimes U^{\otimes n}\right) \times \mathbb{1}_{4} \otimes\left(P_{-} U^{\dagger} \partial_{a} U P_{+} \otimes \mathbb{1}_{4}^{\otimes(n-1)}+\cdots\right)|J, m\rangle \\
& =\frac{1}{2 r}\left(\mathbb{1}_{4} \otimes U^{\otimes n}\right) \times \Gamma^{a} \otimes\left(\Gamma_{a} \otimes \mathbb{1}_{4}^{\otimes(n-1)}+\cdots\right)|J, m\rangle \\
& =\frac{1}{2 r}\left(\mathbb{1}_{4} \otimes U^{\otimes n}\right)\left(\Gamma^{a} \otimes G_{a}^{(n)}\right)|J, m\rangle,
\end{aligned}
$$

where we used (4.45) and (F6) to obtain the second line. Note that the last expression is vanishing as we saw in Sec. IV B 2. Thus, combining these calculations, we find that $\left.\left|\psi_{J m}\right\rangle\right\rangle$ gives a zero eigenvector of the gauge covariant Dirac operator (5.1):

$$
\left.\not D\left|\psi_{J m}\right\rangle\right\rangle=0,
$$

where the gauge field acts as $\left.\left.A\left|\psi_{J m}\right\rangle\right\rangle=\sum_{m^{\prime}}\left|\psi_{J m^{\prime}}\right\rangle\right\rangle(A)_{m^{\prime} m}$.

Let $\{|i\rangle \mid i=1,2, \ldots, N\}$ be any orthonormal basis of $\mathcal{H}_{n}$. By multiplying $\mathbb{1}_{4} \otimes\langle i|$ to the state (5.18), we obtain

$\psi_{i}^{J m}:=\frac{\sqrt{N}}{n+1} \sum_{s=-\frac{1}{2} \gamma=-J+\frac{1}{2}}^{\frac{1}{2}} \sum_{\frac{1}{2} s J-\frac{1}{2} \gamma}^{J-\frac{1}{2}}|1 / 2, s\rangle\left\langle i\left|U^{\otimes n}\right| J-1 / 2, \gamma\right\rangle$.

$\psi_{i}^{J m}$ are $N$ spinors on $S^{4}$, which are also elements of $\operatorname{Ker} \not D$ because of (5.24). We introduce a gauge invariant inner product between these spinors by

$$
\left(\psi_{i}, \psi_{j}\right)=\frac{1}{n+2} \sum_{m=-J}^{J} \frac{3(n+1)^{2}}{8 \pi^{2}} \int d \Omega_{4}\left(\psi_{i}^{J m}\right)^{\dagger} \cdot \psi_{j}^{J m},
$$

where the dot - between $\psi$ 's stands for the contraction of the spinor indices, and $d \Omega_{4}$ is the volume form of the unit $S^{4}$ normalized as $\int d \Omega_{4}=\frac{8 \pi^{2}}{3}$. We multiplied the factor $(n+1)^{2}$ so that the integration measure becomes proportional to the invariant measure made of the information metric. 
Let us calculate $\left(\psi_{i}, \psi_{j}\right)$. By using (5.25), we obtain

$$
\begin{aligned}
\sum_{m}\left(\psi_{i}^{J m}\right)^{\dagger} \cdot \psi_{j}^{J m}= & \frac{N}{(n+1)^{2}} \sum_{m, x, \gamma, \gamma^{\prime}} C_{\frac{1}{2} s J-\frac{1}{2} \gamma}^{J m} C_{\frac{1}{2} s J-\frac{1}{2} \gamma^{\prime}}^{J m} \\
& \times\left\langle j\left|U^{\otimes n}\right| J-1 / 2, \gamma^{\prime}\right\rangle \\
& \times\left\langle J-1 / 2, \gamma\left|U^{\dagger \otimes n}\right| i\right\rangle .
\end{aligned}
$$

By using the summation formula of the Clebsh-Gordan coefficients,

$$
\sum_{\alpha, \gamma} C_{a \alpha b \beta}^{c \gamma} C_{a \alpha b^{\prime} \beta^{\prime}}^{c \gamma}=\frac{2 c+1}{2 b+1} \delta_{b b^{\prime}} \delta_{\gamma \gamma^{\prime}}
$$

we obtain

$$
\begin{aligned}
\sum_{m} \int d \Omega_{4}\left(\psi_{i}^{J m}\right)^{\dagger} \cdot \psi_{j}^{J m}= & \frac{N}{(n+1)^{2}} \frac{2 J+1}{2 J} \\
& \times \int d \Omega_{4}\left\langle j\left|\left(U P_{+} U^{\dagger}\right)^{\otimes n}\right| i\right\rangle .
\end{aligned}
$$

To obtain the last expression, we also used the fact that $|J-1 / 2, \gamma\rangle$ forms a complete basis of $\mathcal{H}_{n}^{+}$and satisfies

$$
\sum_{\gamma}|J-1 / 2, \gamma\rangle\langle J-1 / 2, \gamma|=P_{+}^{\otimes n} .
$$

Finally, by using (E6), we find that (5.29) is given by $\delta_{i j}$ multiplied by a constant factor. Substituting this result into (5.26), we find that

$$
\left(\psi_{i}, \psi_{j}\right)=\delta_{i j}
$$

Namely, $\psi_{i}^{J m}$ are orthonormal under this inner product. Note that, from the index theorem with the second Chern class (4.52), the dimension of $\operatorname{Ker} \not D$ is equal to $N$. Thus, $\left\{\psi_{i}^{J m} \mid i=1,2, \ldots, N\right\}$ gives a complete basis of $\operatorname{Ker} \not D$.

We then consider the Toeplitz quantization map (5.3) for fuzzy $S^{4}$. Note that the orthonormal relation (5.31) implies that the image of the unit constant function on $S^{4}$ is equal to the identity matrix $\mathbb{1}_{\mathcal{H}_{n}}$. In this paper, we assume for simplicity that the function $f$ is gauge singlet, namely, it is proportional to $\delta_{m m^{\prime}}$. In this case, (5.3) can be written more explicitly as

$$
f_{i j}=\frac{3}{8 \pi^{2}} \frac{N}{n+1} \int d \Omega_{4} f\left\langle i\left|\left(U P_{+} U^{\dagger}\right)^{\otimes n}\right| j\right\rangle .
$$

Let us consider the case in which $f$ is the embedding function $x^{A}$ defined in (C9). By using the formula (E7), we find that the image of this embedding function is given as

$$
\hat{x}_{i j}^{A}=\frac{1}{n+4}\left\langle i\left|G_{A}^{(n)}\right| j\right\rangle .
$$

The right-hand side is just the matrix configuration of fuzzy $S^{4}$. Thus, we find that the configuration of fuzzy $S^{4}$ can be obtained as the Toeplitz operator of the embedding function $S^{4} \rightarrow \mathbf{R}^{5}$.

As for the case of $S^{2}$, we can obtain the mapping rules for integrals and the Laplace operator on $S^{4}$. By taking the trace of (5.32), we obtain

$$
\operatorname{Tr} \hat{f}=\frac{3 N}{8 \pi^{2}} \int d \Omega_{4} f .
$$

Thus, integrals are mapped to traces of matrices. Similarly, the image of the Laplace operator on $S^{4}$ is given by

$$
(\hat{\Delta f})_{i j}=-\frac{1}{4 r^{2}}\left[G_{A}^{(n)},\left[G_{A}^{(n)}, \hat{f}\right]\right]_{i j} .
$$

See Appendix G 2 for derivation.

\section{SUMMARY AND DISCUSSION}

In this paper, we developed the notion of the information metric and Berry connection in the context of the matrix geometry. These geometric objects can be defined purely from given matrix configurations and are very useful in characterizing the geometry of matrices. We utilized these objects to see that the well-known matrix configurations of fuzzy $S^{2}$ and fuzzy $S^{4}$ can be viewed in a unified manner as the Toeplitz operators of the embedding functions $S^{n} \rightarrow$ $\mathbf{R}^{n+1}(n=2,4)$. Based on this result, we also obtained mapping rules for the Laplacian on these spaces and found that in both cases, the Laplacian is realized as the matrix Laplacian, $\left[X^{\mu},\left[X^{\mu},\right]\right]$.

The fuzzy $S^{2}$ is the Berezin-Toeplitz quantization such that the gauge group is $U(1)$ and the monopole charge of the connection 1-form is related to the matrix size $N$. The large- $N$ limit corresponds to the limit of large monopole charge. On the other hand, we found that the BerezinToeplitz quantization map for fuzzy $S^{4}$ has a very different structure. The gauge group is non-Abelian and only an $S U$ (2) subgroup has nontrivial gauge connection, which takes the form of the Yang-monopole on $S^{4}$. The Yangmonopole configuration has a fixed instanton number, which is equal to 1 . Thus, the topological charge does not correspond to the matrix size unlike the case of fuzzy $S^{2}$. Instead, the spinors in the quantization map belong to the spin- $J$ representation of the $S U(2)$ subgroup and this spin $J$ is ultimately related to the matrix size of fuzzy $S^{4}$. Thus, the large $N$ limit is not the limit of large instanton number but the limit of the large representation space of spinors.

It would be an interesting problem to construct a different Berezin-Toeplitz quantization on $S^{4}$ such that 
the representation of spinors are fixed but the instanton numbers are given as an increasing sequence. Such map would give a new description of fuzzy $S^{4}$.

What we argued in this paper can be understood as an inverse problem of constructing the Berezin-Toeplitz quantization. In the Berezin-Toeplitz quantization, the matrices (Toeplitz operators) are constructed from the geometric structures such as the metric and gauge field, while we constructed the information metric and Berry connection from the given matrices. For the case of fuzzy $S^{2}$ and fuzzy $S^{4}$, we showed that the $N$-dimensional vector spaces on which the matrices $X^{\mu}$ are acting are indeed identified with the kernel of (differential) Dirac operators, and the associated Berezin-Toeplitz quantization produce $X^{\mu}$ as the Toeplitz operators. This means that our construction indeed gives a solution of the inverse problem. Though we have checked this statement only for $S^{2}$ and $S^{4}$ in this paper, extending this study to more general cases should be important in understanding the geometry of matrices.

The use of the information metric and Berry connection will not be limited only to the same kind of problems of the Berezin-Toeplitz quantization that we considered in this paper. For example, by embedding our setup into systems with D-branes as considered in [22,23], the Berry connection will be identified with the gauge field on D-branes. Through the dualities considered in [28], it will be possible to understand the Seiberg-Witten map for the Berry connection for generic configurations of D-branes. It will also be interesting to see the relation between our findings in this paper and some recent attempts to construct gravitational theories from matrix models [29-31].

The geometric objects presented in this paper are gauge invariant and they provide a new class of observables for matrix models. Though analytic calculation of these observables may be difficult in general, the numerical Monte Carlo method, which has been intensively utilized in studying matrix models recently [32-37], gives a practical way of computing these observables. For some parameter regimes, it will be possible to capture geometric information of matrix models by numerically computing those observables. For example, the weak coupling region of the BMN matrix model [38] on the fuzzy sphere background, which corresponds to the decoupling limit of D2-branes [39], can be studied with this method. However, the interesting regime of the matrix models for M-theory or string theory is not the weak coupling region, unfortunately. We consider that extracting classical geometry by using our observables is not straightforward for such generic sector. This is because the matrix models generically contain higher energy modes than the supergravity and generic configurations of matrices contain contributions from the high energy modes [40-42]. Including those high energy modes, the classical geometric description will not be valid. Thus, we consider that in order to use our observables, it is first needed to find nice degrees of freedom which can probe the low energy physics. (In [41,42], such low energy description was found for a 1/4 BPS sector of the BMN matrix model.) It would be very interesting to find a numerical method of extracting proper low energy degrees of freedom. Such method would also make our observables very useful in studying geometric aspects of the matrix models.

\section{ACKNOWLEDGMENTS}

We thank S. Terashima for valuable discussions on the Berezin-Toeplitz quantization. The work of G. I. was supported, in part, by Program to Disseminate Tenure Tracking System, MEXT, Japan and by KAKENHI (16K17679).

\section{APPENDIX A: INJECTIVITY OF $\rho$ AND $d \rho$}

In this Appendix, we prove the injectivity of $\rho$ and $d \rho$.

We first prove the injectivity of $\rho$ by contradiction. For $y \neq y^{\prime}\left(y, y^{\prime} \in \mathcal{M}\right)$, suppose that $\rho(y)=\rho\left(y^{\prime}\right)$. Since the density matrix is made of zero modes of the Dirac operator, we have

$$
\not D(y) \rho(y)=0 .
$$

From the assumption, we also have

$$
\not D\left(y^{\prime}\right) \rho\left(y^{\prime}\right)=\not D\left(y^{\prime}\right) \rho(y)=0 .
$$

Subtracting (A1) from (A2), we have

$$
\left(\Gamma^{\mu} \otimes\left(y_{\mu}-y_{\mu}^{\prime}\right)\right) \rho(y)=0 .
$$

Similarly, from the right action of the Dirac operator, we also obtain

$$
\rho(y)\left(\Gamma^{\mu} \otimes\left(y_{\mu}-y_{\mu}^{\prime}\right)\right)=0 .
$$

Then, we find that

$$
\begin{aligned}
0 & =\rho(y)\left(\Gamma^{\mu} \otimes\left(y_{\mu}-y_{\mu}^{\prime}\right)\right)\left(\Gamma^{\nu} \otimes\left(y_{\nu}-y_{\nu}^{\prime}\right)\right) \rho(y) \\
& =\frac{1}{2} \rho(y)\left(\left\{\Gamma^{\mu}, \Gamma^{\nu}\right\} \otimes\left(y_{\mu}-y_{\mu}^{\prime}\right)\left(y_{\nu}-y_{\nu}^{\prime}\right)\right) \rho(y) \\
& =\left(y-y^{\prime}\right)^{2} \rho^{2}(y) .
\end{aligned}
$$

As we assumed $y \neq y^{\prime}$, it follows that $\rho(y)=0$. This contradicts with $\operatorname{Tr} \rho(y)=1$. Hence we conclude that $\rho(y) \neq \rho\left(y^{\prime}\right)$ for $y \neq y^{\prime}$, which means that the map $\rho$ is injective.

Next, we show the injectivity of the differential $d \rho$. Let $c^{\mu}(y) \partial_{\mu}$ be a tangent vector field on $\mathcal{M}$ (i.e., $c^{\mu}$ has only tangential components along $\mathcal{M}$ ). We will show below that if $c^{\mu} \partial_{\mu} \rho=0, c^{\mu}$ is vanishing. This is nothing but the injectivity of $d \rho$. Assuming $c^{\mu} \partial_{\mu} \rho=0$ on $\mathcal{M}$, we have 


$$
\begin{aligned}
0 & =c^{\mu}\left(\partial_{\mu} \rho(y)\right)|0, a, y\rangle \\
& =\frac{c^{\mu}}{k}\left(\partial_{\mu} \sum_{b=1}^{k}|0, b, y\rangle\langle 0, b, y|\right)|0, a, y\rangle \\
& =\frac{c^{\mu}}{k}\left(1-\sum_{b=1}^{k}|0, b, y\rangle\langle 0, b, y|\right) \partial_{\mu}|0, a, y\rangle \\
& =\frac{c^{\mu}}{k} \sum_{n \neq 0}|n, y\rangle\left\langle n, y\left|\partial_{\mu}\right| 0, a, y\right\rangle .
\end{aligned}
$$

As $\{|n, y\rangle\}$ is linearly independent, we find that

$$
c^{\mu}\left\langle n, y\left|\partial_{\mu}\right| 0, a, y\right\rangle=0 \quad \text { for } n \neq 0 .
$$

From the relation,

$$
\begin{aligned}
0 & =c^{\mu} \partial_{\mu}(\not D(y)|0, a, y\rangle) \\
& =-c^{\mu} \Gamma_{\mu}|0, a, y\rangle+\not(y) c^{\mu} \partial_{\mu}|0, a, y\rangle,
\end{aligned}
$$

it follows that $c^{\mu}\left\langle n, y\left|\partial_{\mu}\right| 0, a, y\right\rangle=c^{\mu}\left\langle n, y\left|\Gamma_{\mu}\right| 0, a, y\right\rangle / E_{n}$ for $n \neq 0$. Thus, (A7) is equivalent to

$$
c^{\mu}\left\langle n, y\left|\Gamma_{\mu}\right| 0, a, y\right\rangle=0 \quad \text { for } n \neq 0 .
$$

By acting $\langle 0, b, y|$ on the Eq. (A8), we also obtain

$$
c^{\mu}\left\langle 0, b, y\left|\Gamma_{\mu}\right| 0, a, y\right\rangle=0 .
$$

The relations (A9) and (A10) lead to

$$
c^{\mu} \Gamma_{\mu}|0, a, y\rangle=0 .
$$

By using this equation, we can calculate as

$$
\begin{aligned}
0 & =\bar{c}^{\mu} c^{\nu}\left\langle 0, a, y\left|\Gamma_{\mu} \Gamma_{\nu}\right| 0, b, y\right\rangle \\
& =|c|^{2}\langle 0, a, y \mid 0, b, y\rangle \\
& =|c|^{2} \delta_{a b} .
\end{aligned}
$$

This clearly shows that $c^{\mu}=0$. Thus, we have shown that $d \rho$ is an injective map.

\section{APPENDIX B: SPECTRUM OF DIRAC OPERATOR FOR FUZZY $S^{2}$}

In this Appendix, we analyze the spectrum of the Dirac operator for the fuzzy $S^{2}$. We first notice that the Dirac operator (4.6) satisfies

$$
\begin{aligned}
\not^{2}(y)+R \not D(y)= & \left(R^{2} J(J+1)+|y|^{2}\right) \mathbb{1}_{2} \otimes \mathbb{1}_{N} \\
& -2 R\left(y^{i} \frac{\sigma_{i}}{2} \otimes \mathbb{1}_{N}+\mathbb{1}_{2} \otimes y^{i} L_{i}\right) .
\end{aligned}
$$

Consider the operators

$$
\mathcal{O}_{1}(y)=y^{i} \frac{\sigma_{i}}{2} \otimes \mathbb{1}_{N}, \quad \mathcal{O}_{2}(y)=\mathbb{1}_{2} \otimes y^{i} L_{i}
$$

Since the operators $\mathcal{O}_{1}(y), \mathcal{O}_{2}(y)$ and $\not^{2}(y)+R \not D(y)$ mutually commute, they can be simultaneously diagonalized. Thus, the eigenvalue problem of $\not^{2}(y)+R \not D(y)$ is reduced to finding the eigenstates of $\mathcal{O}_{1}(y)$ and $\mathcal{O}_{2}(y)$.

The eigenstates of $y^{i} L_{i}$ can be found as follows. Consider the unitary similarity transformation (C6) which produces the rotation of the vector index. We consider the rotation matrix (C5) with $\alpha=\phi$. In this case, $U$ is explicitly given by (C7) or equivalently by (C8). Under this similarity transformation, $y^{i} L_{i}$ transforms as

$$
y^{i} U^{\dagger} L_{i} U=y^{i}\left(\Lambda^{-1}\right)_{i}^{j} L_{j}=|y| L_{3} .
$$

This implies that the eigenstates of $y^{i} L_{i}$ are give by $U|J, m\rangle$, where $|J, m\rangle$ is the standard basis defined in (4.4). Note that $U$ depends only on the angular variables for $y$.

Diagonalizing $y^{i} \frac{\sigma_{i}}{2}$, which appears in $\mathcal{O}_{1}(y)$, is just the spacial case of the above argument such that the dimension of the representation is equal to 2 . Thus, its eigenstates are given by $U|1 / 2, \pm\rangle$. Thus, the simultaneous eigenstates of $\mathcal{O}_{1}(y)$ and $\mathcal{O}_{2}(y)$ are given by

$$
U_{2}(y)|1 / 2, \pm\rangle \otimes U_{N}(y)|J, m\rangle
$$

where the subscripts of $U_{2}$ and $U_{N}$ just stand for the dimensions of the representation spaces on which they are acting.

(B4) gives the eigenstates of $\not^{2}(y)+R \not D(y)$. For each eigenstate, the eigenvalue of $\not^{2}(y)+R \not D(y)$ is given by

$$
R^{2} J(J+1)+|y|^{2}-2 R|y|(m \pm 1 / 2) .
$$

Note that the states $U_{2}(y)|1 / 2,+\rangle \otimes U_{N}(y)|J, J\rangle$ and $U_{2}(y)|1 / 2,-\rangle \otimes U_{N}(y)|J,-J\rangle$ are not degenerate but the other states are doubly degenerate.

The nondegenerate eigenstates of $\not^{2}(y)+R \not D(y)$ are also eigenstates of $\not(y)$ itself. Thus, we find the following eigenstates of $\not(y)$ :

$$
\begin{aligned}
& \left|\psi_{J}\right\rangle=\left(U_{2} \otimes U_{N}\right)|1 / 2,+\rangle \otimes|J, J\rangle, \\
& |\psi \cdot\rangle=\left(U_{2} \otimes U_{N}\right)|1 / 2,-\rangle \otimes|J,-J\rangle .
\end{aligned}
$$

For the degenerate eigenstates of $\not^{2}(y)+R \not(y)$, we generally need to take a linear combination of them to find the eigenvectors of $\not{D}(y)$. Thus, we consider

$$
\begin{aligned}
\left|\psi_{m}\right\rangle= & \left(U_{2} \otimes U_{N}\right)\left(a_{m}|1 / 2,+\rangle \otimes|J, m\rangle\right. \\
& \left.+b_{m}|1 / 2,-\rangle \otimes|J, m+1\rangle\right)
\end{aligned}
$$

for $m=-J,-J+1, \ldots, J-1$. By acting the Dirac operator on these states, we obtain 


$$
\begin{aligned}
\not\left|\psi_{m}\right\rangle= & \left(U_{2} \otimes U_{N}\right)\left[R \sigma^{3} \otimes L^{3}-\sigma^{3} \otimes|y|\right. \\
& \left.+\frac{R}{2}\left(\sigma_{+} \otimes L_{-}+\sigma_{-} \otimes L_{+}\right)\right] \\
& \times\left(a_{m}|1 / 2,+\rangle \otimes|J, m\rangle+b_{m}|1 / 2,-\rangle\right. \\
& \otimes|J, m+1\rangle),
\end{aligned}
$$

where we utilized the properties of the unitary matrices $U_{2}$ and $U_{N}$ shown in Appendix $\mathrm{C} 1$. The action of the $S U(2)$ generators on the right-hand side can also be explicitly computed by using (4.4). Assuming that $\left|\psi_{m}\right\rangle$ are eigenstates of $\not(y)$ with eigenvalues $\lambda_{m}$, we can obtain the following equations for $a_{m}$ and $b_{m}$ :

$$
\lambda_{m}\left(\begin{array}{l}
a_{m} \\
b_{m}
\end{array}\right)=\left(\begin{array}{cc}
R m-|y| & R \sqrt{(J-m)(J+m+1)} \\
R \sqrt{(J-m)(J+m+1)} & -R(m+1)+|y|
\end{array}\right)\left(\begin{array}{l}
a_{m} \\
b_{m}
\end{array}\right) .
$$

The characteristic equation reads

$$
\begin{gathered}
0=\operatorname{det}\left(\begin{array}{cc}
R m-|y|-\lambda_{m} & R \sqrt{(J-m)(J+m+1)} \\
R \sqrt{(J-m)(J+m+1)} & -R(m+1)+|y|-\lambda_{m}
\end{array}\right) \\
=\lambda_{m}^{2}+R \lambda_{m}-|y|^{2}+R(2 m+1)|y|-R^{2} J(J+1) .
\end{gathered}
$$

The eigenvalues are then given by

$$
2 \lambda_{m}^{( \pm)}=-R \pm \sqrt{R^{2}+4\left\{|y|^{2}-R(2 m+1)|y|+R^{2} J(J+1)\right\}} .
$$

The corresponding coefficients $a_{m}^{( \pm)}$and $b_{m}^{( \pm)}$have to satisfy (4.9) to give a solution of (B10). The normalization condition for the state $\left|\psi_{m}\right\rangle$ also imposes (4.10). Note that, without loss of generality, both $a_{m}^{( \pm)}$and $b_{m}^{( \pm)}$can be set to be real numbers. Thus, the two Eqs. (4.9) and (4.10) fully determine the states $\left|\psi_{m}\right\rangle$.

\section{APPENDIX C: REPRESENTATION MATRICES OF SPECIAL UNITARY GROUPS}

\section{Representation matrices of $S O(3)$}

In this Appendix, we explicitly write down representation matrices of a $S O(3)$ rotation which transforms a general unit vector into the unit vector pointing the north pole.
Let $x$ be a general vector in $R^{3}$ parametrized as

$$
\left(\begin{array}{c}
x^{1} \\
x^{2} \\
x^{3}
\end{array}\right)=\left(\begin{array}{c}
\sin \theta \cos \phi \\
\sin \theta \sin \phi \\
\cos \theta
\end{array}\right),
$$

and $x_{0}$ be the unit vector pointing the North pole as

$$
\left(\begin{array}{l}
x_{0}^{1} \\
x_{0}^{2} \\
x_{0}^{3}
\end{array}\right)=\left(\begin{array}{l}
0 \\
0 \\
1
\end{array}\right) .
$$

We can consider an $S O(3)$ rotation which transforms $x$ to $x_{0}$,

$$
x^{i}=\left(\Lambda^{-1}\right)_{i j} x_{0}^{j} .
$$

$\Lambda^{-1}$ is explicitly given by

$$
\begin{aligned}
\Lambda^{-1} & =\left(\begin{array}{ccc}
\cos \theta \cos \phi & -\sin \phi & \sin \theta \cos \phi \\
\cos \theta \sin \phi & \cos \phi & \sin \theta \sin \phi \\
-\sin \theta & 0 & \cos \theta
\end{array}\right)\left(\begin{array}{ccc}
\cos \alpha & \sin \alpha & 0 \\
-\sin \alpha & \cos \alpha & 0 \\
0 & 0 & 1
\end{array}\right) \\
& =\left(\begin{array}{ccc}
\cos \phi & -\sin \phi & 0 \\
\sin \phi & \cos \phi & 0 \\
0 & 0 & 1
\end{array}\right)\left(\begin{array}{ccc}
\cos \theta & 0 & \sin \theta \\
0 & 1 & 0 \\
-\sin \theta & 0 & \cos \theta
\end{array}\right)\left(\begin{array}{ccc}
\cos \alpha & \sin \alpha & 0 \\
-\sin \alpha & \cos \alpha & 0 \\
0 & 0 & 1
\end{array}\right) .
\end{aligned}
$$


Note that in defining $\Lambda^{-1}$ there is an ambiguity of $S O(2)$ rotations around the north pole. This ambiguity is represented by the angle $\alpha$.

Now, let us consider the action of this rotation on the generators of $S O(3)(S U(2))$. Let $L^{i}(i=1,2,3)$ be any irreducible representation matrices of $S U(2)$ generators. Since the representation matrices of the generators of Lie algebra are invariant tensors, there always exist unitary similarity transformations which undo the rotation of the vector index. Thus, there exists a unitary matrix $U$ satisfying

$$
U^{\dagger} L_{i} U=\left(\Lambda^{-1}\right)_{i j} L_{j} .
$$

If $\Lambda^{-1}$ is the 3-dimensional (vector) representation matrix of an element $g$ of $S U(2)$, the unitary matrix $U$ is given by the $N$-dimensional irreducible representation of the same element $g$, where $N$ is the dimension of the representation of $L^{i}$.

Below, we fix the ambiguity in the definition of $\Lambda^{-1}$ by putting $\alpha=\phi$. From (C5), we find that the unitary matrix $U$ satisfying (C6) is given by

$$
U=e^{-i \phi L_{3}} e^{-i \theta L_{2}} e^{i \phi L_{3}} .
$$

This has another expression:

$$
U=e^{z L^{-}} e^{-L^{3} \log \left(1+|z|^{2}\right)} e^{-\bar{z} L^{+}},
$$

where $L^{ \pm}=L^{1} \pm i L^{2}$ and we introduced the stereographic coordinate $(z, \bar{z})$ defined in (4.13).

\section{Representation matrices of $S O(5)$}

In this Appendix, we show representation matrices of $S O(5)$ rotations.

Let us first consider a unit vector in $\mathbf{R}^{5}$, which can be parametrized in the polar coordinate as

$$
\left(\begin{array}{l}
x^{1} \\
x^{2} \\
x^{3} \\
x^{4} \\
x^{5}
\end{array}\right)=\left(\begin{array}{c}
\sin \theta \sin \phi \sin \psi \sin \chi \\
\sin \theta \sin \phi \sin \psi \cos \chi \\
\sin \theta \sin \phi \cos \psi \\
\sin \theta \cos \phi \\
\cos \theta
\end{array}\right)
$$

We also consider the unit vector $x_{0}$ pointing the North pole given by

$$
\left(\begin{array}{l}
x_{0}^{1} \\
x_{0}^{2} \\
x_{0}^{3} \\
x_{0}^{4} \\
x_{0}^{5}
\end{array}\right)=\left(\begin{array}{l}
0 \\
0 \\
0 \\
0 \\
1
\end{array}\right)
$$

There exists $S O(5)$ rotation which transforms $x_{0}$ to $x$ as

$$
x^{A}=\Lambda_{B}^{A} x_{0}^{B} .
$$

This transformation can be written as a product of some $S O(2)$ rotations. Indeed, $\Lambda$ is given by a composition of a rotation on the 5-4 plane with angle $\theta$, a rotation on the 4-3 plane with angle $\phi$, a rotation on the 3-2 plane with angle $\psi$ and finally a rotation on the 2-1 plane with angle $\chi$.

We will write down the explicit form of $\Lambda$ in the following. We introduce the generators of $S O(5)$ Lie algebra, $\Sigma_{A B}, A, B \in\{1,2,3,4,5\}$, which satisfies

$\left[\Sigma_{A B}, \Sigma_{C D}\right]=\delta_{A D} \Sigma_{B C}+\delta_{B C} \Sigma_{A D}-\delta_{A C} \Sigma_{B D}-\delta_{B D} \Sigma_{A C}$.

The fundamental (vector) and the spinor representation matrices of $\Sigma_{A B}$ are given by

$$
\begin{aligned}
D_{\mathrm{V}}\left(\Sigma_{A B}\right)_{C D} & =\delta_{A C} \delta_{B D}-\delta_{A D} \delta_{B C}, \\
D_{\mathrm{S}}\left[\Sigma_{A B}\right] & =\frac{1}{2} \Gamma_{A B}=\frac{1}{4}\left[\Gamma_{A}, \Gamma_{B}\right],
\end{aligned}
$$

respectively. For example, in the vector representation, $\Sigma_{54}$ can be written as

$$
D_{\mathrm{V}}\left(\Sigma_{54}\right)=\left(\begin{array}{ccccc}
0 & & & & \\
& 0 & & & \\
& & 0 & & \\
& & 0 & -1 \\
& & & 1 & 0
\end{array}\right) .
$$

This generates the rotation on the 5-4 plane with angle $\theta$,

$$
D_{\mathrm{V}}\left(e^{-\theta \Sigma_{54}}\right)=\left(\begin{array}{ccccc}
1 & & & & \\
& 1 & & & \\
& & 1 & & \\
& & \cos \theta & \sin \theta \\
& & & -\sin \theta & \cos \theta
\end{array}\right) .
$$

Then, the rotation matrix $\Lambda$ in $(\mathrm{C} 11)$ can be represented as

$$
\Lambda=D_{\mathrm{V}}\left(e^{-\chi \Sigma_{21}} e^{-\psi \Sigma_{32}} e^{-\phi \Sigma_{43}} e^{-\theta \Sigma_{54}}\right) .
$$

The spinor representation of the same group element,

$$
\begin{aligned}
U & =D_{\mathrm{S}}\left(e^{-\chi \Sigma_{21}} e^{-\psi \Sigma_{32}} e^{-\phi \Sigma_{43}} e^{-\theta \Sigma_{54}}\right) \\
& =e^{-\chi \Gamma_{21} / 2} e^{-\psi \Gamma_{32} / 2} e^{-\phi \Gamma_{43} / 2} e^{-\theta \Gamma_{54} / 2}
\end{aligned}
$$

satisfies the relation

$$
\Lambda_{A B} \Gamma_{B}=U^{\dagger} \Gamma_{A} U .
$$




\section{APPENDIX D: HAMILTONIAN METHOD}

\section{Hamiltonian method for fuzzy $S^{2}$}

In this Appendix, we compute the classical geometry of $S^{2}$ by using the Hamiltonian method.

The Hamiltonian for the fuzzy $S^{2}$ configuration (4.1) is given by

$H(y)=\frac{1}{2}\left(R L^{i}-y^{i}\right)^{2}=\frac{1}{2}\left(R^{2} J(J+1)+|y|^{2}\right)-R y^{i} L_{i}$.

Thus, the problem is just reduced to diagonalizing the operator $y^{i} L_{i}$. This is done in Appendix B, and the eigenstates are given by $U(y)|J, m\rangle$, where $U(y)$ is the $N$-dimensional representation matrix of the $S O(3)$ rotation defined in Appendix $\mathrm{C} 1$. The eigenvalues of $H(y)$ are given by

$$
\frac{1}{2}\left(R^{2} J(J+1)+|y|^{2}\right)-R m|y| .
$$

In particular, the ground state is given by $m=J$. In the large$N$ limit, the ground state energy converges to

$$
\frac{1}{2}(1-|y|)^{2} .
$$

The classical geometry is defined as zeros of this function. Thus, we find that the classical geometry is given by a unit sphere,

$$
\mathcal{M}=\left\{y \in \mathbf{R}^{3}|| y \mid=1\right\} .
$$

The information metric and the Berry connection can also be computed in the similar way to the case of the Dirac operator. By using the differential of the ground state (4.14), one can quickly check that in the large- $N$ limit, the information metric and Berry connection are equal to those obtained in Sec. IVA 3.

\section{Hamiltonian method for fuzzy $S^{4}$}

The Hamiltonian for the matrices (4.24) is given by

$$
H(y)=\frac{1}{2}\left(1+|y|^{2}\right)-y_{A} X^{A}+\mathcal{O}(1 / n),
$$

where $|y|=y_{A} y^{A}$. In order to find the spectrum of this Hamiltonian, we consider the specific $S O(5)$ rotation matrix $\Lambda$ that brings the vector in the direction of the pole $(0,0,0,0,|y|)$ to the position vector of a point $y \in \mathbf{R}^{5}$ : $y_{A} \Lambda_{B}^{A}=|y| \delta_{B 5}$. As shown in Appendix C 2, for this rotation there exists a corresponding unitary operator $U$ which satisfies (C18). It follows from the relation (C18) that

$$
U^{i \otimes n}\left(y_{A} X^{A}\right) U^{\otimes n}=|y| X_{5} .
$$

Using this relation we can diagonalize the Hamiltonian as
$H(y)=U^{\otimes n}\left[\frac{1}{2}\left(1+|y|^{2}\right)-|y| X_{5}+\mathcal{O}(1 / n)\right] U^{\dagger \otimes n}$.

Then we can easily find the ground states of $H(y)$ as

$\left|0^{(\alpha)}, y\right\rangle=U^{\otimes n}|n / 2, n / 2-\alpha\rangle \quad \alpha \in\{0,1, \ldots, n\}$.

Here, the notation $|J, m\rangle$ introduced in Sec. IV B 2 is used on the right-hand side. Note that $J=(n+1) / 2$ in Sec. IV B 2, while $J=n / 2$ in this Appendix. This difference comes from the fact that the Dirac orator is defined in a bigger vector space. The eigenvalue of the ground states is

$$
E_{0}(y)=\frac{1}{2}\left(1-|y|^{2}\right)+\mathcal{O}(1 / n) .
$$

In the classical limit, the zeros of $E_{0}(y)$ are points such that $|y|=1$, and the classical space is indeed $S^{4}$ with unit radius.

Note that the structure of the ground state is common to that in the Dirac operator method. Hence, in the large- $N$ limit, the Berry connection and the information metric for the Hamiltonian method are equivalent to those in the Dirac operator method.

\section{APPENDIX E: DERIVATION OF USEFUL RELATIONS FOR FUZZY $S^{4}$}

In this Appendix, we prove some useful relations for fuzzy $S^{4}$.

We first prove (4.26). We first calculate as

$$
\sum_{A}\left(G_{A}^{(n)}\right)^{2}=5 n \mathbb{1}_{\mathcal{H}_{n}}+2 \mathcal{O} .
$$

Here, $\mathcal{O}$ is given by

$$
\mathcal{O}=\Gamma_{A} \otimes \Gamma_{A} \otimes \mathbb{1}_{4} \otimes \mathbb{1}_{4}+\cdots,
$$

where $\cdots$ stands for all the symmetric permutations of the positions of $\Gamma_{A}$ 's in the first term (i.e., $\mathcal{O}$ has totally $n(n-1) / 2$ terms). It is easy to see that $\mathcal{O}$ commutes with all of the $S O(5)$ generators, (4.23). Thus, from Schur's lemma, $\mathcal{O}$ is proportional to the identity matrix on $\mathcal{H}_{n}$. The normalization constant can be fixed by acting $\mathcal{O}$ on the vector $\left|\eta_{1}\right\rangle^{\otimes n}$. By using the representations (4.22), we can easily prove that

$$
\sum_{a=1}^{4}\left(\Gamma^{a} \otimes \Gamma^{a}\right)\left|\eta_{1}\right\rangle \otimes\left|\eta_{1}\right\rangle=0 .
$$

Then, we obtain

$$
\mathcal{O}\left|\eta_{1}\right\rangle^{\otimes n}=\frac{n(n-1)}{2}\left|\eta_{1}\right\rangle^{\otimes n} .
$$

Hence, we find that

$$
\mathcal{O}=\frac{n(n-1)}{2} \mathbb{1}_{\mathcal{H}_{n}} .
$$

Substituting this into (E1), we obtain (4.26). 
Next, we prove the following equations:

$$
\begin{gathered}
\int d \Omega_{4}\left(U P_{+} U^{\dagger}\right)^{\otimes n}=\frac{16 \pi^{2}}{(n+2)(n+3)} \mathbb{H}_{\mathcal{H}_{n}}, \\
\int d \Omega_{4} x_{A}\left(U P_{+} U^{\dagger}\right)^{\otimes n}=\frac{16 \pi^{2}}{(n+2)(n+3)(n+4)} G_{A}^{(n)} .
\end{gathered}
$$

Here, the volume form $d \Omega_{4}$ shall be normalized as $\int d \Omega_{4}=$ $\frac{8 \pi^{2}}{3}$ and $x_{A}$ in the second equation is defined in (C9). These equation follow from the fact that the integrations over $S^{4}$ produce only rotationally invariant tensors. Thus, from the structures of indices, we can see that the right-hand sides of (E6) and (E7) are proportional to the identity matrix and $G_{A}^{(n)}$, respectively ${ }^{8}$ Namely, we have

$$
\begin{gathered}
\int d \Omega_{4}\left(U P_{+} U^{\dagger}\right)^{\otimes n}=\alpha \mathbb{1}_{\mathcal{H}_{n}}, \\
\int d \Omega_{4} x_{A}\left(U P_{+} U^{\dagger}\right)^{\otimes n}=\beta G_{A}^{(n)} .
\end{gathered}
$$

The remaining task is to determine the proportionality constants $\alpha$ and $\beta$. $\alpha$ is determined by taking the trace of the both sides in (E8). Noting that

$\operatorname{Tr}_{\mathcal{H}_{n}}\left(U P_{+} U^{\dagger}\right)^{\otimes n}=\operatorname{Tr}_{\mathcal{H}_{n}} P_{+}^{\otimes n}=\operatorname{Tr}_{\mathcal{H}_{n}^{+}} \mathbb{1}_{\mathcal{H}_{n}}=\operatorname{dim} \mathcal{H}_{n}^{+}=n+1$,

we find that $\alpha$ is given as in (E6). $\beta$ is determined by multiplying $G_{A}^{(n)}$ and taking a summation over $A$ and finally taking the traces in the both sides of (E9). Because of (4.26), the right-hand side of (E9) becomes

$\beta n(n+4) \operatorname{Tr}_{\mathcal{H}_{n}} \mathbb{1}_{\mathcal{H}_{n}}=\beta \frac{n(n+1)(n+2)(n+3)(n+4)}{6}$.

Because of (C11) and (C18), the left-hand side of (E9) becomes

\footnotetext{
${ }^{8}$ Note that any contractions of the vector indices of Gamma matrices as in (E2) give the trivial identity matrix as shown in (E5).
}

$$
\begin{aligned}
& \int d \Omega_{4} x^{A} \Lambda_{A B} \operatorname{Tr}_{\mathcal{H}_{n}}\left(G_{B}^{(n)} P_{+}^{\otimes n}\right) \\
& =\int d \Omega_{4} \operatorname{Tr}_{\mathcal{H}_{n}}\left(G_{5}^{(n)} P_{+}^{\otimes n}\right) \\
& =\int d \Omega_{4} \operatorname{Tr}_{\mathcal{H}_{n}^{+}}\left(G_{5}^{(n)}\right)=\frac{8 \pi^{2}}{3} n(n+1) .
\end{aligned}
$$

By equating (E11) and (E12), we finally obtain (E7).

\section{APPENDIX F: SPIN CONNECTIONS ON $S^{2}$ AND $S^{4}$}

In this Appendix, we list the spin connections on $S^{2}$ and $S^{4}$.

\section{Spin connections on $S^{2}$}

The standard round metric on $S^{2}$ in the stereographic coordinate is given by

$$
d s^{2}=r^{2} \frac{d z d \bar{z}}{\left(1+|z|^{2}\right)^{2}},
$$

where $r$ is any positive constant corresponding to the radius of the sphere. We introduce the vielbein by

$$
e^{+}=\frac{r d z}{1+|z|^{2}}, \quad e^{-}=\frac{r d \bar{z}}{1+|z|^{2}},
$$

so that $d s^{2}=e^{+} e^{-}$. The spin connection $\omega$ is determined by the equations $d e^{\alpha}+\omega_{\beta}^{\alpha} \wedge e^{\beta}=0$. In our case, the equations reduce to

$$
\begin{aligned}
\omega^{+}{ }_{+} \wedge e^{+} & =\frac{z}{r} e^{-} \wedge e^{+}, \\
\omega^{-} \wedge e^{-} & =\frac{\bar{z}}{r} e^{+} \wedge e^{-} .
\end{aligned}
$$

The solution to these equations is given by

$$
\omega^{+}+=-\omega_{-}^{-}=\frac{1}{r}\left(z e^{-}-\bar{z} e^{+}\right)=\frac{z d \bar{z}-\bar{z} d z}{1+|z|^{2}} .
$$

\section{Spin connections on $S^{4}$}

The standard round metric on $S^{4}$ in the polar coordinate is given by

$$
\begin{aligned}
d s^{2}= & r^{2}\left(d \theta^{2}+\sin ^{2} \theta d \phi^{2}+\sin ^{2} \theta \sin ^{2} \phi d \psi^{2}\right. \\
& \left.+\sin ^{2} \theta \sin ^{2} \phi \sin ^{2} \psi d \chi^{2}\right),
\end{aligned}
$$

where $r$ is the radius of $S^{4}$. We define the vielbein by

$$
\begin{aligned}
& e^{1}=r \sin \theta \sin \phi \sin \psi d \chi, \\
& e^{2}=r \sin \theta \sin \phi d \psi, \\
& e^{3}=r \sin \theta d \phi, \\
& e^{4}=r d \theta .
\end{aligned}
$$

By solving the equations $d e^{\alpha}+\omega_{\beta}^{\alpha} \wedge e^{\beta}=0$, we obtain 
the spin connection as

$$
\begin{aligned}
& \omega_{12}=\cos \psi d \chi, \\
& \omega_{13}=\cos \phi \sin \psi d \chi, \\
& \omega_{14}=\cos \theta \sin \phi \sin \psi d \chi, \\
& \omega_{23}=\cos \phi d \psi, \\
& \omega_{24}=\cos \theta \sin \phi d \psi, \\
& \omega_{34}=\cos \theta d \phi .
\end{aligned}
$$

\section{APPENDIX G: QUANTIZATION MAPS FOR LAPLACIANS ON $S^{2}$ AND $S^{4}$}

In this Appendix, we derive the mapping rules (5.15) and (5.35) for Laplace operators on $S^{2}$ and $S^{4}$, respectively.

\section{Laplacian on $S^{2}$}

Here, we derive (5.15). From the mapping rule (5.12), we have

$(\hat{\Delta f})_{i j}=\left(\mathcal{D}_{a}^{2} \psi_{j}, f \psi_{i}\right)+\left(\psi_{j}, f \mathcal{D}_{a}^{2} \psi_{i}\right)+2\left(\mathcal{D}_{a} \psi_{j}, f \mathcal{D}_{a} \psi_{i}\right)$,

where we have used partial integrations. The first and the second terms in (G1) can be evaluated with the formula

$$
\mathcal{D}_{a}^{2} \psi_{i}=-\frac{J}{r^{2}} \psi_{i}
$$

This is obtained as follows. Since $\not \supset \psi_{i}=0$, we have

$$
\begin{aligned}
\mathcal{D}_{a}^{2} \psi_{i} & =\left(\sigma^{a} \sigma^{b}-\sigma^{a b}\right) \mathcal{D}_{a} \mathcal{D}_{b} \psi_{i} \\
& =-\frac{1}{2} \sigma^{a b}\left[\mathcal{D}_{a}, \mathcal{D}_{b}\right] \psi_{i} \\
& =-\frac{1}{2} \sigma^{a b}\left(\frac{1}{4} R_{a b c d} \sigma^{c d}-i F_{a b}\right) \psi_{i} .
\end{aligned}
$$

For $S^{2}$ with radius $r$, the curvature tensor is given by

$$
R_{a b c d}=\frac{1}{r^{2}}\left(\delta_{a c} \delta_{b d}-\delta_{a d} \delta_{b c}\right),
$$

and $F_{a b}=e_{a}^{\mu} e_{b}^{\nu} F_{\mu \nu}$ is obtained from (4.17) as

$$
F_{12}=\frac{N}{2 r^{2}} \text {. }
$$

Substituting (G4) and (G5) into (G3), we obtain (G2). The third term in (G1) is evaluated by using

$$
\mathcal{D}_{ \pm} \psi_{i}=\frac{1}{r} \Lambda_{\mp k}\left(L_{k}\right)_{i j} \psi_{j} .
$$

These equations follow from (5.6) and (C6). By using the relation

$$
\sum_{a=1}^{2} \Lambda_{a k} \Lambda_{a k^{\prime}}=\delta_{k k^{\prime}}-x^{k} x^{k^{\prime}},
$$

where $x^{k}$ is defined in (C1), we find that the third term in (G1) is given by

$$
-\frac{2 J^{2}}{r^{2}} \hat{f}_{i j}+\frac{2}{r^{2}}\left(L_{k} \hat{f} L_{k}\right)_{i j}
$$

From this and (G3), we obtain (5.15).

\section{Laplacian on $S^{4}$}

The mapping rule for the Laplace operator on $S^{4}$ can be obtained in a similar way as the case of $S^{2}$. First, it is easy to see that

$$
\frac{1}{2} \Gamma^{a b} F_{a b}^{k}=\frac{4 i}{r^{2}} D_{S}\left(J^{k}\right) .
$$

The curvature tensor of $S^{4}$ with radius $r$ is given by the same form as (G4), where the indices $a, b, c, d$ run from 1 to 4 for $S^{4}$. Then, from (G9), the relation

$$
\Gamma^{a b}\left[D_{a}, D_{b}\right] \psi_{i}^{J m}=\frac{4 J-2}{r^{2}} \psi_{i}^{J m}
$$

holds. From (5.25), it is also easy to obtain

$D_{a} \psi_{i}^{J m}=\frac{\sqrt{N}}{2 r(n+1)} \sum_{s, \gamma} C_{\frac{1}{2} s J-\frac{1}{2} \gamma}^{J m}|1 / 2, s\rangle\left\langle i\left|U^{\otimes n} G_{a}^{(n)}\right| J-1 / 2, \gamma\right\rangle$.

By using (G10) and (G11), we can evaluate the Toeplitz operator for the Laplacian on $S^{4}$ defined by

$\widehat{\Delta f}_{i j}=\frac{1}{n+2} \sum_{m} \frac{3(n+1)^{2}}{8 \pi^{2}} \int d \Omega_{4}\left(\psi_{j}^{J m}\right)^{\dagger} \psi_{i}^{J m} \Delta f$.

By integrating by parts, this is given by the sum of terms such as $\int d \Omega_{4}\left(\psi_{j}^{J m}\right)^{\dagger}\left(D_{a}^{2} \psi_{i}^{J m}\right) f, \int d \Omega_{4}\left(D_{a}^{2} \psi_{j}^{J m}\right)^{\dagger} \psi_{i}^{J m} f$ and $\int d \Omega_{4}\left(D_{a} \psi_{j}^{J m}\right)^{\dagger}\left(D_{a} \psi_{i}^{J m}\right) f$. The first two can be evaluated by noting that

$$
\begin{aligned}
D_{a} D_{a} \psi_{i}^{J m} & =\left(\Gamma^{a} \Gamma^{b}-\Gamma^{a b}\right) D_{a} D_{b} \psi_{i}^{J m} \\
& =-\frac{1}{2} \Gamma^{a b}\left[D_{a}, D_{b}\right] \psi_{i}^{J m},
\end{aligned}
$$

and using (G10), while the third term can be calculated with (G11). These calculations lead to the mapping rule (5.35). 
[1] T. Banks, W. Fischler, S. H. Shenker, and L. Susskind, Phys. Rev. D 55, 5112 (1997).

[2] N. Ishibashi, H. Kawai, Y. Kitazawa, and A. Tsuchiya, Nucl. Phys. B498, 467 (1997).

[3] B. de Wit, J. Hoppe, and H. Nicolai, Nucl. Phys. B305, 545 (1988).

[4] J. Arnlind, J. Hoppe, and G. Huisken, J. Diff. Geom. 91, 1 (2012).

[5] J. Madore, Classical Quantum Gravity 9, 69 (1992).

[6] A. P. Balachandran, B. P. Dolan, J. H. Lee, X. Martin, and D. O'Connor, J. Geom. Phys. 43, 184 (2002).

[7] A. Connes, M. R. Douglas, and A. S. Schwarz, J. High Energy Phys. 02 (1998) 003.

[8] J. Arnlind, M. Bordemann, L. Hofer, J. Hoppe, and H. Shimada, J. High Energy Phys. 06 (2009) 047.

[9] J. Castelino, S. Lee, and W. Taylor, Nucl. Phys. B526, 334 (1998).

[10] M. Sperling and H. C. Steinacker, J. Phys. A 50, 375202 (2017).

[11] M. Bordemann, E. Meinrenken, and M. Schlichenmaier, Commun. Math. Phys. 165, 281 (1994).

[12] X. Ma and G. Marinescu, J. Geomet. Anal. 18, 565 (2008).

[13] K. Hasebe, SIGMA 6, 071 (2010).

[14] K. Hasebe, Int. J. Mod. Phys. A 31, 1650117 (2016).

[15] K. Hasebe, arXiv:1712.07767.

[16] G. Ishiki, Phys. Rev. D 92, 046009 (2015).

[17] L. Schneiderbauer and H. C. Steinacker, J. Phys. A 49, 285301 (2016).

[18] D. Berenstein and E. Dzienkowski, Phys. Rev. D 86, 086001 (2012).

[19] M. H. de Badyn, J. L. Karczmarek, P. Sabella-Garnier, and K. H. C. Yeh, J. High Energy Phys. 11 (2015) 089.

[20] J. L. Karczmarek and K. H. C. Yeh, J. High Energy Phys. 11 (2015) 146.

[21] G. Ishiki, T. Matsumoto, and H. Muraki, J. High Energy Phys. 08 (2016) 042.
[22] T. Asakawa, G. Ishiki, T. Matsumoto, S. Matsuura, and H. Muraki, arXiv:1804.00161.

[23] S. Terashima, arXiv:1804.00647.

[24] S. Terashima, J. High Energy Phys. 10 (2005) 043.

[25] T. Asakawa, S. Sugimoto, and S. Terashima, J. High Energy Phys. 03 (2002) 034.

[26] K. Hashimoto and S. Terashima, J. High Energy Phys. 02 (2006) 018.

[27] Z. Guralnik and S. Ramgoolam, J. High Energy Phys. 02 (2001) 032.

[28] N. Seiberg and E. Witten, J. High Energy Phys. 09 (1999) 032.

[29] Y. Kaneko, H. Muraki, and S. Watamura, Classical Quantum Gravity 35, 055009 (2018).

[30] H. C. Steinacker, J. High Energy Phys. 12 (2016) 156.

[31] M. Hanada, H. Kawai, and Y. Kimura, Prog. Theor. Phys. 114, 1295 (2005).

[32] Y. Asano, V. G. Filev, S. Kovacik, and D. O'Connor, arXiv:1805.05314.

[33] K. Hatakeyama, A. Tsuchiya, and K. Yamashiro, arXiv:1805.03975.

[34] E. Berkowitz, E. Rinaldi, M. Hanada, G. Ishiki, S. Shimasaki, and P. Vranas, Phys. Rev. D 94, 094501 (2016).

[35] D. Kadoh and S. Kamata, arXiv:1503.08499.

[36] S. W. Kim, J. Nishimura, and A. Tsuchiya, Phys. Rev. Lett. 108, 011601 (2012).

[37] S. Catterall and T. Wiseman, J. High Energy Phys. 04 (2010) 077.

[38] D. E. Berenstein, J. M. Maldacena, and H. S. Nastase, J. High Energy Phys. 04 (2002) 013.

[39] J. M. Maldacena, M. M. Sheikh-Jabbari, and M. Van Raamsdonk, J. High Energy Phys. 01 (2003) 038.

[40] J. Polchinski, Prog. Theor. Phys. Suppl. 134, 158 (1999).

[41] Y. Asano, G. Ishiki, S. Shimasaki, and S. Terashima, Phys. Rev. D 96, 126003 (2017).

[42] Y. Asano, G. Ishiki, S. Shimasaki, and S. Terashima, J. High Energy Phys. 02 (2018) 076. 